\title{
HOMER ALPHA 1
}

Since Christian August Lobeck it had been established as a matter of fact that «Orpheus» was a post-Homeric invention of the «Orphics» and that all poetry handed down under the name of Orpheus was a forgery and falsification borrowed from the verses of Homer and Hesiod. In order to reach a decision in this question of priority the following paper is divided into two distinct parts. Part one deals with expressions and poetical elements of the so-called proem of the Iliad, showing that with regard to the analysis (in its form given to it by P. Von der Mühll) the proem is part of the work of the last «homeric poet, the composer of the Iliad and Odyssey in the form in which we have them. Part two is based on the author's studies on Orpheus and the pre-Homeric epic tradition and the result attained is that Orpheus was a singer of the Mycenaean World. As a consequence of this the author was finally in a position to identify the last Homeric poet as a member

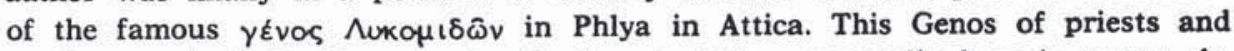
soothsayers had of old a genuine Orpheus-tradition, especially hymnic poetry devoted to Demeter. This result of the second part agrees with that one of the first; that the proem as part of the last poet's work is composed of second-hand poetry. Thence it emerges that the ancient tradition, underlying Hippolytos' and Tzetzes' assertion, is the right one and cannot be overthrown in favour of a modern -but meanwhile obsolete- theory.

DIS MANIBVS $\mathbf{V} \cdot \mathbf{D} \cdot \mathbf{M}$

Verwegen könnte es erscheinen, eine schon anderthalb Jahrhunderte akzeptierte und eingewurzelte philologische Lehrmeinung mit neuen Gründen durch eine den antiken Uberzeugungen angemessenere Auffassung ersetzen zu wollen. Doch ist es an der Zeit, dass dies nun endlich geschieht, nachdem jetzt alle Voraussetzungen dazu gegeben sind.

Der die Ilias eröffnende Vers ist allgemein als 'homerisch' bekannt:

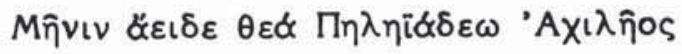

Daneben kannte griechische Uberlieferung einen 'Orpheus'-Vers:

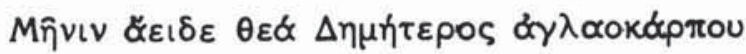


(Orph. fr. 48). Antike Uberzeugung war es, dass der 'Homer'-Vers nach dem 'Orpheus'-Vers geschaffen sei ${ }^{1}$. Die moderne Philologie hingegen ist sich seit Christian August Lobeck einig, dass es umgekehrt gewesen sei. "Ein Blick auf die orphischen Verse zeigt, dass sie wie natürlich mit homerischen Formeln arbeiten, dazu aber in perfidester Weise plagiieren, um ihrerseits Homer als Plagiator zu kennzeichnen; dafür ist der schlagendste Beweis die Korrektur, die die Orphiker des 6. Jahrhunderts dem Demeterhymnus, dessen Verse sie benutzen, geben...; wir haben weiter dieselbe Erscheinung in fr. 48 Kern und dem Anfange der Ilias" ${ }^{2}$... "wie sich die Orphiker ja auch zu der Behauptung verstiegen, dass der Anfangsvers der Ilias dem orphischen Verse... nachgebildet $\operatorname{sei}^{3}{ }^{3} .$. " the opening line of a poem on the 'wrath of Demeter', adapted from Il. I 1 ${ }^{4}$. Von Lobeck bis Richardson, also von 1829 bis 1974, besteht Einmütigkeit im Urteil über die beiden 'Menis-Verse'. Ein Skandalfall im frühesten Griechentum also, wenn die modernen Behauptungen von der "Behauptung" der 'Orphiker' zutreffen: ein Skandalfall der Modernen freilich, wenn deren Anschuldigung unzutreffend und ungerechtfertigt sein sollte. Und man hat inzwischen allen Grund zur Skepsis gegenüber der modernen Theorie. Zwei Gegebenheiten sind es, die heute zu einer Revision des Falles nötigen. Erstens, weil die unter des Orpheus Namen überlieferten Dichtungspassagen des Demeterhymnus alle, ausnahmslos, eine ältere und ursprünglichere Version darstellen als die unter Homers Namen stehende Demeterdichtung ${ }^{5}$, sodass die beigezogene Parallele bereits hinfällig und die 'selbstverständliche' Priorität Homers nicht mehr gegeben ist. Und zweitens, weil Orpheus selbst inzwischen nicht mehr eine in nachhomerischer Zeit 'erfundene' Archegetengestalt, eine «zu Repristinationszwecken» 'zurechtgemachte' Legende ist, sondern - der antiken Uberlieferung konform- eine Sängergestalt mittelmykenischer Zeit ${ }^{6}$, sodass die These

1 Iustinus, Cohort. ad gentiles 17 b (p. 66 Otto). Ebenso Tzetzes Exeg. in Iliad., p. 26 Hermann. S. Kern, Orphic. Frgm., 1922, fr. 48.

2 Lobeck, Aglaoph. II SS. 591 f., 827 ff., beidemal ohne Ausserung zur Priorität: man hat sie wohl indirekt dem Cap. III zu entnehmen, bes. II 255 ff., 317 «Orphicae... fabulae telam... uno fortasse et altero post Homerum saeculo coeptam et paullo ante Onomacritum absolutam esse», - Zitat: Geffcken, «Antike Kulturkämpfe», N. Jbb. 29, 1912, S. 596, 3 (die Frgm.-Nr. nach Abel ist im Zitat durch die nach Kern ersetzt).

3 K. Ziegler, $R E$ 18, 2, 1396, 29 ff. (erschienen 1942).

4 N. J. Richardson, The Homeric Hymn to Demeter, Oxford 1974, S. 78 (dazu Th. Gelzer, Mus. Helv. 34, 1977, S. 249). Zahlreiche andere könnten angefügt werden.

5 Zunächst nur vom Motivischen gezeigt: G. Murray, The Rise of the Greek Epic, 1911, S. 350 ff. Dann umfassend: R. Böhme, Orpheus, 1970, Kap. III (S. 75 ff.). Ders., Peisistratos und sein homerischer Dichter, 1983, SS. 16-24, bes. 20 f.

6 Marmor Parium A 25 ep. 14, Diels-Kr. Vs. 1 B 15. R. Böhme, Sänger der 
der 'orphischen Plagiatdichtung' aus Homer und Hesiod zumindest in Frage gestellt ist. Beides schafft eine völlig neue Situation: der Fall des $I l$. I 1 ist neu aufzurollen.

Grundlagen zur Beurteilung des Falles sind gegeben, da ja beide Verse nicht allein stehen sondern jeweils einem Zusammenhang angehören: der 'Homer'-Vers dem Ilias-Proömium, der 'Orpheus'-Vers dem Demetermythos und =hymnus, den es zumindest doppelt gibt, nämlich als Kleinepos unter Homers Namen und in Form von Zitationen einprägsamer Passagen aus 'Orpheus'-Dichtung ?. Beides lässt sich heute beurteilen. Unsere Darlegung wird aus zwei Teilen bestehen: einem ersten, in dem die beiden konkurrierenden Verse in ihrem jeweiligen Zusammenhang der Kritik unterzogen werden, und einem zweiten, in dem über den Dichter des Ilias-Proömiums zu sprechen sein wird.

I

$\mathrm{Zu}$ beginnen ist mit der sprachlichen Form, in der der spät bezeugte

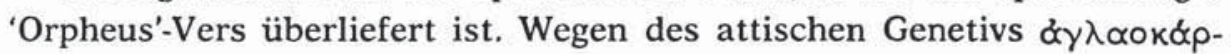
tou wird mancher wohl dazu neigen, den Vers vorschnell für 'jung' und folglich für sekundär zu halten. Der Wert dieses Arguments wird aber sofort klar, wenn man nur einmal annimmt, vom 'homerischen' Demeterhymnus sei zufällig durch Zitation nur gerade der vierte Vers erhalten:

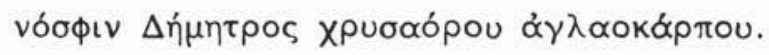

Der gleiche attische Genetiv vom gleichen Epitheton im gleichen Zusammenhang des Demetermythos würde zu einem ganz entsprechenden Fehlurteil verleiten, obwohl dieser selbe Genetiv -zudem noch im Verein mit einem zweiten, xpuoðópou- viele nicht davon abgehalten hat, den mit diesem Vers überlieferten Hymnus ins 7. Jahrhundert zu datieren. Dieser Genetiv kann also auch im Menis-Vers nicht anders beurteilt werden als innerhalb des 'homerischen' Hymnus: ein Indiz für die Prioritätsfrage zwischen 'Orpheus' und 'Homer' ist er auf keinen Fall. Und das genügt zunächst, um ein allfälliges Vorurteil abzuweisen.

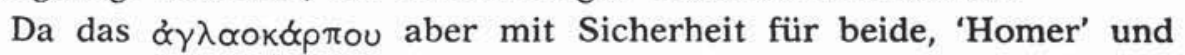
'Orpheus', nicht sehr alt sein kann, wird dies Epitheton wohl ein älteres

Vorzeit, 1980, SS. 32-52; ders., Minos 17, 1981, S. 122 ff.; ders., Peisistratos, SS. 13-15, $61-75,108 \mathrm{f}$.

7 Es gab mehrere Versionen, s. Orpheus, S. 101 ff.; Richardson. S. 74 ff. 
ersetzen. Darauf führt der überlieferte Hymnus selbst ${ }^{8}$. In dessen Vers 315 ,

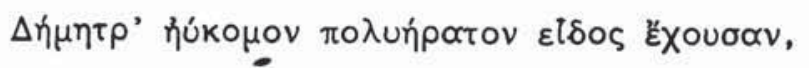

ist der zweite Halbvers ein überkommenes Element. Er hat nämlich eine wörtliche Entsprechung im Hesiod (Th. 908) und ist auch dort keine hesiodeische Erfindung oder Eigenschöpfung zur Charakterisierung der Okeanostochter Eurynome sondern ein auch jenem bereits geläufiges poetisches Element, das als 'homerisch'-hesiodeische Konkordanz - wie inzwischen ausreichend abgesichert ist ${ }^{9}$ - ein alttradiertes poetisches Orpheus-Element darstellt. Dazu fügt sich ja auch der $\mathrm{Zu}$ sammenhang, dass nämlich im vorangehenden Vers (314) das

$$
\text { ТI }
$$

durch seine wiederholte Wiederkehr beim Il. VIII-Dichter (v. 398, daneben XI 185) gleichfalls der gleichen Orpheustradition zuzuweisen ist: der unselbständige Il. VIII-Dichter ist ja, wo er nicht Homer in Kontribution setzt, als Orphikos erwiesen ${ }^{10}$, sodass im Demeterhymnus also nicht zweierlei zusammengestückt ist, ein Halbvers aus Hesiod mit einem dem It. VIII entnommenen Element ${ }^{11}$, sondern poetische Elemente aus ein und derselben Tradition zum Kontext gefügt sind. Darum dürfte im Zusammenhang dieser Verse auch das Zwischenglied $\Delta \eta \mu \eta \tau \rho$ '

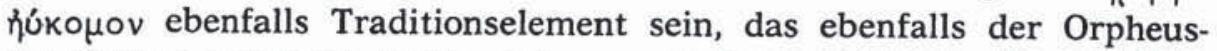
Tradition entstammt. Dahin weisen auch die Wiederkehr des gleichen Elements im Anfangsvers des 'homerischen' Hymnus (v. 1) sowie noch die Wiederholung des Demeterepitheton rúkopos in gleicher Position im Vers 297, wo der Name der Göttin, durch die Casusvariante bedingt, dahinter treten musste. Kein Zweifel also: wie die Umgebung ist auch

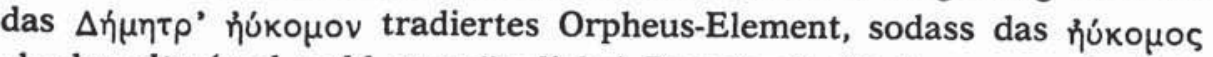
als das alte (und wohl ursprüngliche) Demeter-Epitheton anzusehen ist.

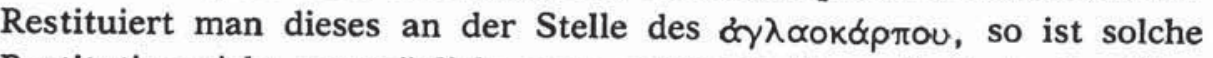
Restitution nicht nur möglich: man erhält als ältere Variante den Vers

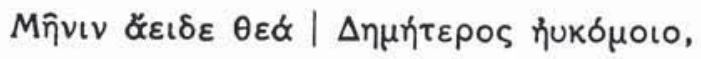

8 Mit der hier folgenden Argumentation zum Epitheton vgl. Orpheus, S. 80 f.

9 Orpheus, SS. 35-80, bes. Sänger $d$. Vz., SS. 63-71, auch Peisistratos, S. 19 f.

10 Für seine Unselbständigkeit s. ex. gr. Leaf, Introd. to Il. VIII. Als Orphikos: Peisistratos, SS. 29-38.

11 Gegen diese unvorstellbare Annahme eines Zusammenstückens entlegenster Teil-Elemente hat sich schon Hoekstra gewandt, Mnemosyne 10, 1957, S. $193 \mathrm{ff}$. 
dessen zweite Hälfte im Hinblick darauf, dass auch der 'Orpheus - Hypoklept' Hesiod diese bereits kennt -

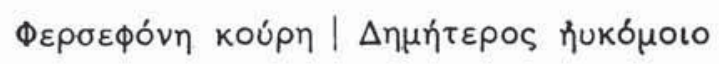

(fr. 280, $20 \mathrm{MW}$ ) - als altorpheisches Hemistich gesichert ist: auch

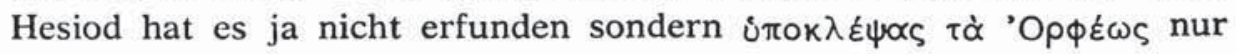
benutzt ${ }^{12}$. Und das Gleiche tat ja ebenso der Letzte Dichter in einer seiner Ilias-Partien:

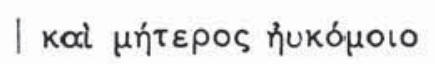

(Il. XXIV 466) ${ }^{13}$. - Die sehr späten Zeugen, die uns den Orpheus-Vers von der Demeter Menis anführen, konnten ihn in ihrer Zeit natürlich längst nicht mehr in seiner alten Form kennen: im Blick auf die stetige Fortentwicklung der 'Orpheus'-Dichtungen in historischer Zeit ${ }^{14}$ sollte das selbstverständlich sein. Besonders jung braucht die spätbezeugte Version darum noch nicht zu sein, da ja (wie zu Beginn gesagt) auch der 'homerische' Demeterhymnus dies sekundäre Epitheton mit seinem attischen Genetiv schon enthält.

Doch nun zurück zur Prioritätsfrage, für die es auf der 'Orpheus'-

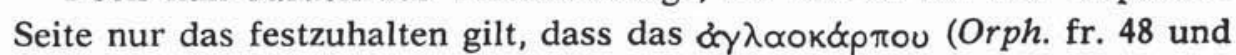
'Hom.' Hy. Dem. 4) in neutram partem etwas zu beweisen vermag.

Anders steht es mit dem Ilias-Proömium. Unitarier mögen in dessen Eröffnungsvers den ältesten Vers der abendländischen Literatur $=$ und Geistesgeschichte bewundern: andere werden sachlicher und sachbezogener urteilen. Das sogenannte 'Proömium' gehört nämlich nach seiner Form und Zweckbestimmung nicht unter die alten - kitharodischen$\pi \rho o o l \mu l \alpha$, welche Götterhymnen waren ${ }^{15}$, sondern unter jene AödenUberleitungen, mit denen die Sänger nach vorangegangenem hymnischen Prooimion den Ubergang zu nachfolgender erzählender Dichtung bewerkstelligten. Als Hinleitung und Hinweis auf das was folgt nennt Eusthatios die Anfangspartie der Ilias darum richtiger «Proekthesis»16

12 Des Hesiod 'Orpheus-Hypoklepsie': schol. Lycophr. 399, p. 149, 13 Scheer (Kern test. 246), wozu emritis 19, 1951, S. 107 ff. Als zutreffend erwiesen Sänger d. $V z$., S. $16 \mathrm{ff}$. und $63 \mathrm{ff}$. (vgl. auch Orpheus, S. 49, 5).

13 Noch Archestratos (4. Jh.) folgt dem alten Versschluss huxópoio in seinem

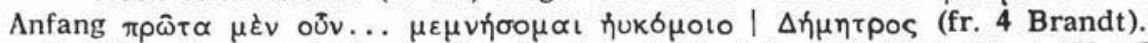

14 Diese Fortentwicklung seit etwa dem 5. Jh. behandelt M. L. West, The Orphic Poems, Oxford 1983.

15 Pind., Nem. II 1. Thuk., III 104 mit schol. Plut., de mus. 4, 1132 D und 6, 1133 C. Aristid., or. 34 p. 245 K., u. a. m.: die gesamten 24 Zeugnisse mit kurzer Besprechung bei R. Böhme, Das Prooimion, Bühl/Baden 1937, SS. 11-24.

16 Eusthat. ad Iliad. I, p. 7, 44 ff. ed. Rom., 7, 37 ff. ed. Lips. 1827. 
(während der gängige Begriff 'Proömium' im Sinne von «Einleitung» ein nicht vor dem 4. Jahrhundert gebräuchlicher metaphorischer Terminus ist). Und als 'Proekthesis' zur nachfolgenden Ilias reiht sich diese Einleitung in ihr Genos d.h. in eine ganze Reihe anderer derartiger Uberleitungen ein, von denen auch uns noch etwa zwei Dutzend bekannt sind ${ }^{17}$. Diese müssen hier nicht nochmals ausgeschrieben werden. Besieht man sie sich in ihrer Struktur und Funktion genauer, so ist jedenfalls deutlich, dass a lle (ursprünglich also auch die im SchriftEpos einem Kontext eingefügten) den Vorhergang eines richtigen Prooimion d.h. eines Götterhymnus voraussetzen, um danach zu erzählender Dichtung überzuleiten ${ }^{18}$. Von der Regel weichen einzig vier formal jüngere $a b$, die lediglich auf die folgende Dichtung hinleiten, ohne dass der Vorhergang eines hymnischen Prooimion auch nur denkbar wäre: so bei Ilias, Thebais, Ilias parua, Odyssee ${ }^{19}$. Diese sind ersichtlich zu 'Literatur' gewordene Spätformen dessen, was vorher eher gewohnheitsmässige Improvisation des Aöden war, sind also etwas durchaus Sekundäres, aus dem alten Brauch Erwachsenes, also kein 'episches Urgestein'. Derartiges kann erst mit der schriftlichen Aufzeichnung des tradierten Epos Gestalt angenommen haben.

Verweist dieser Sachverhalt die Einleitungen zu Ilias und Odyssee in die späte Zeit der Redaktion der Homerika zu den beiden Grossepen, so geben sie selbst diese ihre späte Entstehung sowie das Vorliegen epischer Sekundärdichtung auch deutlich genug zu erkennen. Vor allen konkreten Einzelheiten ist bereits das zu bedenken: beide wollen ja eine Eröffnung und Hinleitung auf das Gesamtepos sein: eine Einleitung zur Gesamt-Ilias oder Gesamt-Odyssee kann aber nicht vor der Konzeption des jeweiligen Gesamtplans entstanden sein. Diese Einleitungen sind also zwangsläufig das Werk des 'Redaktors', des Schöpfers der jeweiligen dispositio totius operis. Hinter dessen grossem Planen bleibt -wie immer beim attischen Redaktor- die dichterische Ausführung zurück. So ist denn auch diejenige zur Odyssee, gerade weil sie eine solche zur ganzen Odyssee sein will, schon im vorigen Jahrhundert von I. Bekker kritisch gewürdigt worden, und diese Kritik «besteht in jedem Satz zu Recht, allem apologetischen Gezappel zum Trotz» ${ }^{20}$.

17 Il. XI 218-220. XIV 508-510. XVI 112 f. Aristoxenos in Anecd. Osanni (Nauck, Lex. Vindob., p. 273), usw.: die Texte (mit kurzen Erläuterungen) s. Orpheus, S. 20 f.

18 Paradigma: Musenhymnus - Uberleitung - Theogonie, so im überlieferten Hesiod. So auch in der sogen. Ilias des Apellikon, s. Aristoxenos in voriger Anm.

19 S. Orpheus, a. O. Nr. VII, VIII, XIV, XVII.

20 I. Bekker, SBA $1853=$ Homerische Blätter I, S. 69 ff. Das diese Kritik stützende Zitat von Ed. Schwartz, Odyssee, 1924, S. 288, 1. 
Doch kann das Odyssee-'proömium' für unsere Frage ganz bei Seite bleiben. Die mit dem Menis-Vers anhebende Einleitung zur Ilias zeigt ebenso alles, worauf es hier ankommt: ihre späte Entstehung, ihre Zugehörigkeit zum poetischen Anteil des 'Redaktors' der Gesamt-Ilias, ihre Unoriginalität.

Zunächst steht die Ilias-Einleitung nicht konkurrenzlos allein. Es gab eine andere in der 'Ilias des Apellikon': in ihr wird noch nach altem Brauch mit dem Vorhergang eines Hymnus gerechnet (der An-

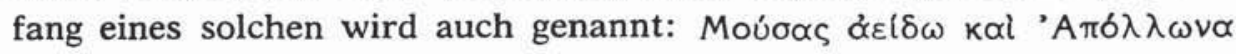
$\kappa \lambda \cup \tau \delta \tau \circ \xi \circ \nu$ ), woran sich dann eine kurze Uberleitung schloss:

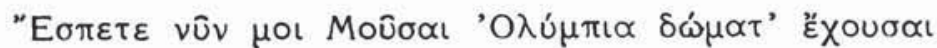

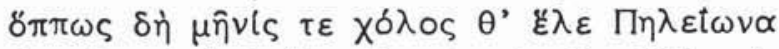

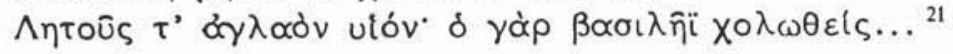

Das ist also eine Uberleitung zu Il. I 9 ff.: aber eben nur zur Ursache vom Zorn des Peliden und Groll des Apollon, also nicht zur gesamten Ilias. Während diese mit dem bekannten 'Proömium' konkurrierende Uberleitung somit nur einen kleinen Geschehnisabschnitt ins Auge fasst, ist die der Vulgat-Ilias darauf ausgerichtet, den grossen Zusammenhang des überlieferten Grossepos -zumindest aller vor die eigentliche Achilleis (Il. XIX ff.) fallenden Geschehnisse- einzuleiten. Das kann nur das Werk dessen sein, der unter Benutzung des Zorn-Motivs - das ihm (in anderer sprachlicher Ausdrucksweise) zwar vorgegeben war ${ }^{22}$, aber auch erlaubte, den ersten Helden auf weite Strecken zurückzustellen-den grossen Gesamtzusammenhang geschaffen hat ${ }^{23}$, kann also nur das Werk des das Ganze redigierenden Letzten Dichters sein.

Als solches erweist sich diese Einleitung nun auch in konkreten Einzelheiten. So ist schon festgestellt, dass das $\tau \dot{\alpha} \pi \rho \omega \hat{\tau} \alpha$ in Vers 6 mit seinem Artikel zu den 'jungen' Ausnahmeerscheinungen der epischen Dichtersprache gehört ${ }^{24}$. Sieht man nun, dass solches adverbiales $\tau \dot{\alpha}$ $\pi \rho \hat{\omega} \tau \alpha$ mitsamt der gleichwertigen Variante $\tau \dot{\partial} \pi \rho \hat{\tau} \tau o v$ im gesamten 'homerischen' Epos sich zwar noch 27 mal findet, dass alle diese Stellen

${ }^{21}$ Zum Anecd. Osanni s. Das Prooimion, S. 13 f.

$z$ Vorgegeben als $\chi 0 \lambda \omega \theta \hat{\eta} v \alpha \iota$ bezw. $\chi \propto \lambda \lambda \varepsilon \pi \hat{\imath} \nu \alpha \iota$, z. B. Il. I 9, XVIII 111, 108,

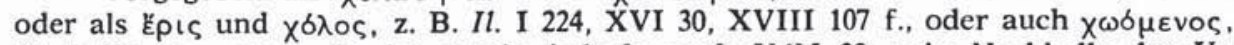
Il. I 429, das zwar "B» ist, worin jedoch, nach VdM 29, «ein Nachhall» des Ursprünglichen zu sehen ist. (Vorhomerisch, in der alten Memnonis, war es ja eine mütterliche Warnung, die den Achill sich vom Kampf zurückhalten liess, Schadewaldt, Von Homers Welt und Werk, SS. 167, $181 \mathrm{ff}$.$) .$

${ }_{23}$ So richtig Cauer, in der zweiten Aufl., S. $508 \mathrm{f}$. Vgl. Bethe III, S. $46 \mathrm{f}$.

24 M. Leumann, Homerische Wörter (= Schweiz. Beitr. z. Altwiss. 3), 1950, S. 12. Vgl. auch Orpheus, S. 21 ff. Nr. XVIII und XIX, wozu 27, 3, sowie Emped., Fr. 62, 4. 
nach der Analyse jedoch dem Letzten Dichter gehören ${ }^{25}$, so wird man dessen Vorkommen im Introitus doch wohl im Rahmen dieses Befundes zu beurteilen und folglich auch hier an die Hand des Letzten Dichters zu denken haben. Und da es sich nicht als sekundär erweisen oder durch etwas Alteres ersetzen lässt, ist allein damit das Urteil über das ganze Proömium gefällt -ein Urteil über die Entwicklungsstufe, der es zugehört (was noch lange kein Qualitätsurteil ist).

Sodann zeigt der Ilias-Anfang weitere bemerkenswerte Elemente, die ihn unmittelbar der - gleichfalls späten, gleichfalls dem Letzten Dichter gehörigen - Nekyia-Dichtung verbinden. Das der Schattenseele entgegen-

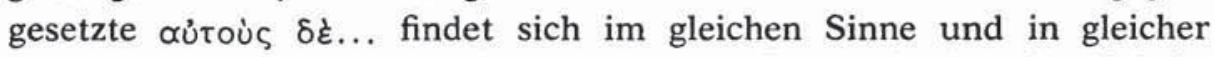
metrischer Position auch in jenem seltsamen Passus, in dem der NekyiaDichter den Herakles als Schatten im Hades weilen lässt, diesem sogleich aber dessen "Selbst» entgegensetzt:

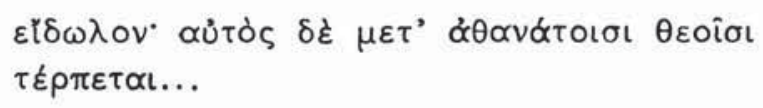

(Od. XI 602 ff.). Das ist die Kunst des gleichen Letzten Dichters: die gleiche Vorstellungswelt bei gleicher poetischer Ausdrucksweise.

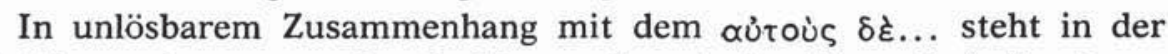
Einleitung der vorangehende Vers mitsamt der Besonderheit, die er enthält:

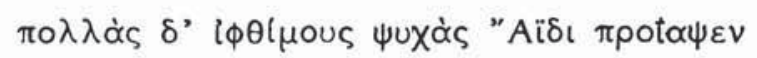

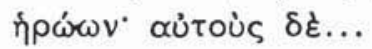

Das ist keine originale Dichtung. Denn dieser Vers findet sich mit leichter Variante sowohl im Anfang des Il. XI (54 f.):

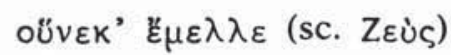

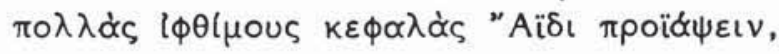

als auch bei Hesiod (fr. 204, 116 ff. MW):

$$
\begin{aligned}
& \varepsilon \tilde{\mu} \varepsilon \lambda \lambda \varepsilon v \ldots
\end{aligned}
$$

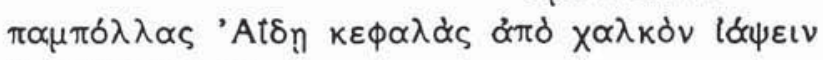

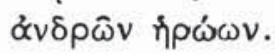

25 Die Stellen: Il. IV 267, 424, VI 489, XII 420, XIII 679, XVII 612, XXIII 275 , $324,523,538$, Od. I 257 , IV $13,159,509$, VIII 268, 553, XIV 467, XXIII 214, H. Dem. 86, Ap. 71, 214, 407, 493, Herm. 428, 487, Aphr. 179, 185. Alle Il.= und Od. -Stellen gehören dem Letzten Dichter, s. jeweils VdM ad ll., was lediglich einmal, $I l$. VI 489, übersehen ist: $487 \mathrm{fin}$. wie XI 55 (s. gleich), also de eingeschobene Reflexion 487. $89=« \mathrm{~B} »$. Im Od. XXIII schliesst 225 an 212 an, 213-17 ist auch Vorbereitung zu 218 ff. $\mathrm{Zu}$ den Hy. s. Anfang von Teil II. 
Wenn dabei das $(\pi \alpha \mu) \pi \circ \lambda \lambda \alpha \grave{s} \kappa \varepsilon \phi \propto \lambda \grave{\alpha} \varsigma$ dem Il. XI und dem Hesiod gemeinsam ist, so kann kein Zweifel darüber aufkommen, dass in diesem das Ursprüngliche vorliegt, während in der Einleitung die $\kappa \varepsilon \phi \alpha \lambda \alpha$ l durch die $\psi$ ux $\alpha$ l ersetzt sind ${ }^{20}$, die 'Köpfe' durch die 'Seelen', die in den Hades geschickt werden: eine Änderung zu sekundärer Ausdrucksweise, die sich dem Dichter darum auferlegt hat, weil die Köpfe ja zu den den Hunden und Vögeln vorgeworfenen Leibern d.h. zu den "đủтol" gehören. Diese 'gescheite' - d.h. rationalistisch glättendeAnderung hat nun aber eine von ihrem Autor nicht bedachte Folge: im Gegensatz zur sonstigen 'homerischen' Auffassung von den kraftlosen Schattenseelen gibt es jetzt die Seltsamkeit der $\lceil\phi \theta \iota \mu \circ \iota \quad \psi v \chi \alpha \uparrow$ -natürlich nur an dieser einen Stelle. Da nun sowohl die Ersetzung der $\kappa \varepsilon \phi \alpha \lambda \alpha \propto$ durch die $\psi u \chi \alpha$ wie auch der Grund dieser Anderung und zudem auch die 'oddity' des Ergebnisses evident sind, lässt sich das Verhältnis des Il. I 3 zur ursprünglicheren poetischen Wendung keinesfalls umkehren. Und das führt unausweichlich zu folgendem Sachverhalt: Da ja Il. XI 54 f. zweifelsfrei dem Letzten Dichter gehört ${ }^{27}$, ist es zwangsläufig dieser selbst, der die ihm geläufige poetische Wendung auch in $\mathrm{Il}$. I 3 -und hier in der abgewandelten Form- benutzt hat ${ }^{28}$. Nach dem $\tau \dot{\alpha} \pi \rho \hat{\omega} \tau \alpha$ und dem $\alpha \dot{\tau} \tau o \dot{\varsigma} \varsigma \delta \dot{\varepsilon}$ wird man damit zum dritten Mal auf den Letzten Dichter als den Schöpfer des Introitus geführt. Und am besprochenen Fall bleibt bemerkenswert: nur wenn man sowohl das Urbild als auch den Grund der Anderung kennt, erhält das Besondere und Befremdliche dieser Dichtung seine Aufklärung. Das wird in dieser Einleitung nicht der einzige derartige Fall bleiben.

Neben dem sachlich, sprachlich und metrisch gleichen Versanfang

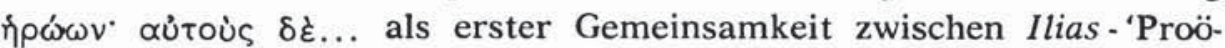
mium' und Nekyia ist in der einleitenden Partie noch von einem Ratschluss des Zeus die Rede:

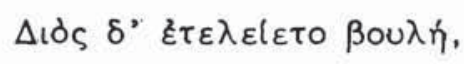

der ebenfalls in der Nekyia wörtlich wiederkehrt (Od. XI 297). Auch das ist keine originale Dichtung: die Wendung entstammt den Kyprien und ist an beiden Stellen vom Letzten Dichter nur benutzt. Denn in den Kyprien ${ }^{29}$ war der Gedanke ausgeführt, dass Zeus mit der von

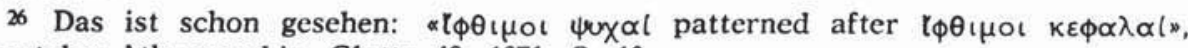
Apostolos Athanassakis, Glotta 49, 1971, S. 13.

27 S. Leaf, Introd. to Il. XI, VdM, S. 191.

28 Nicht nur Il. I 3 sondern ebenso VIII 487 fin. gehört zu XI 55, s. vorletzte Anm.

29 Cypria fr. 1 (p. 118 Allen). 
Menschen überfüllten Erde Mitleid gehabt habe, dass er daher bei sich nachgedacht und beschlossen habe, die Mutter Erde dadurch zu erleichtern, dass er den Brand des grossen Krieges unter die Menschen schleudere, und so wurden viele Helden vor Troia getötet und des Zeus Ratschluss erfüllt. Es ist kein Zweifel —und ja auch schon gesehen ${ }^{30}$ das die Wendung da, wo von einem Ratschluss des Zeus expressis verbis erzählt wird, primär ist gegenüber dem Ilias-Anfang, wo man ihren Sinn nur versteht, wenn man des Zeus Absicht, das Kyprienmotiv, bereits kennt. Und man versteht dann auch, «dass die Worte in der Ilias, wo sie etwas inkonzinn an die voraufgehenden, grammatisch parallelen Relativsätze - in Parenthese- angeschlossen sind, nicht so glatt in den Zusammenhang eingefügt sind ${ }^{31}$. Somit ist auch die Scho-

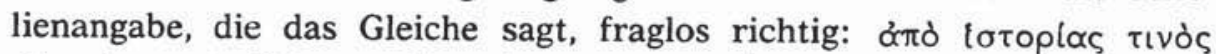

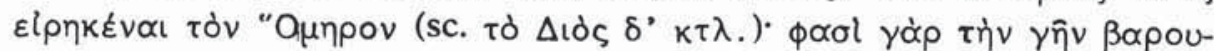
$\mu \varepsilon^{\prime} v \eta v$ ú $\pi^{\prime} \alpha^{2} v \theta \rho \dot{\omega} \pi \omega \nu \pi 0 \lambda \cup \pi \lambda \eta \theta \varepsilon l \alpha \varsigma$ usw. ${ }^{32}$. Die Ratschlussformel des Ilias-Introitus ist also den Kyprien entlehnt. Und nicht anders verhält es sich mit ihr in der Nekyia. Zu deren Heroinenkatalog (in dem sie sich findet) hat nämlich Wilamowitz schon früh festgestellt, dass Leda, die als Mutter der Dioskuren nicht aber der Helena genannt wird, auf die Kyprien führe - denn dort war Helena Tochter des Nemesis-, womit ihm feststand "dass die Nekyia aus den Kyprien schöpft» ${ }^{33}$. Es ist also der späte Letzte Dichter, der beidemal, in seiner Ilias-Einleitung wie in seiner Nekyia, im Aufblick zu den Kyprien gedichtet und daraus die Ratschlussformel übernommen hat ${ }^{34}$.

Sieht man auch hier wieder die Sekundärdichtung, die Applikation einer vorgegebenen poetischen Wendung, so erklärt sich gleich eine weitere Seltsamkeit des Introitus. Achills Zorn habe viele kräftige Achäerseelen in den Hades geschickt, sie selbst aber den Hunden und Vögeln zur Beute gemacht. Nun ist es nicht seltsam, von Hunden und Vögeln gefressen zu werden, das gibt es auch sonst: "es wird ihm

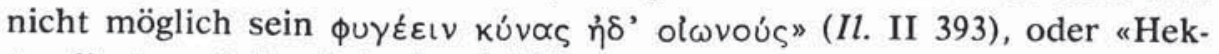

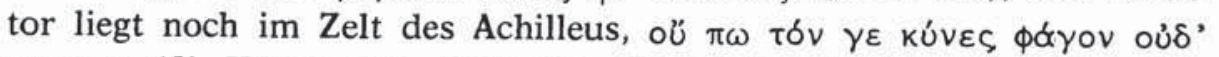
ol $\omega v o$ " (Il. XXIV 411). Und das gibt es in gleicher oder ähnlicher

\footnotetext{
30 So z. B. von Kullmann, Philologus 99, 1955, S. $186 \mathrm{f}$.

31 So Kullmann a. O.

32 Schol. AD ad Il. I 5. Bethe, Hom., II, SS. 154 f., 228. Kullmann, a. O., SS. 167 ff. und 100, 1956, S. 132 f. Webster, From Myc. to Homer, 1958, S. 180 f.

33 Wilamowitz, Hom. Unters. (Philol. Unters. 7. Heft, 1884), S. 149.

34 Schon für VdM ist ja der Letzte Dichter $\approx \mathrm{B} *$ für Ilias und Odyssee der gleiche: z. B. Hypomnema, SS. 10, 18, 59, 140 ff., 204, 351, 374, und er spricht vom "jüngeren Dichter, der auch sonst Berührung mit den Kyprien zeigt», a. O., S. 14.
} 
Form noch oft ${ }^{35}$. Das in der Proekthesis Seltsame ist die Aussage, dass der Zorn Achills die vielen Achäer den Hunden und a 11 en Vögeln zur Beute gemacht habe. Ist nun die Ratschlussformel aus den Kyprien übernommen und als fertiges poetisches Element nur eingefügt, so ist evident, dass in dieser unoriginalen Dichtung das fragwürdige $\pi \tilde{\alpha} \sigma \iota$ nur ein wenig geschicktes Füllsel ist, um die erborgte Formel anfügen zu können ${ }^{36}$. Und d.h. abermals: weiss man um das Vorbild, das dieser Dichtung des Redaktors zu Grunde liegt, so versteht man auch hier wieder ihre Besonderheit.

Es gibt in der Proekthesis noch zwei weitere Besonderheiten, auf die das Gleiche zutreffen dürfte: dass sie nur vom Vorbild her verständlich sind. Wenn etwas im Epos uraltertümlich ist, so ist es der häufige Beisatz des Patronymikon zum individuellen Personennamen, und zwar entweder in der Form eines patronymischen Namens oder als Genetiv des Vatersnamens (mit oder ohne "Sohn"). Uberall wo auf die eine oder andere Weise der Vater mitgenannt wird, kommt dessen Name - wie das in der Natur der Sache liegt- erst als zwe it es Glied. Dieser Gebrauch ist völlig konstant ${ }^{37}$. Doch der erste Iliasvers hat die seltsame Voranstellung des Patronymikon, die umso mehr nach Erklärung verlangt, als das Metrum dem üblichen Gebrauch nicht im Wege gestanden hätte ${ }^{38}$. Und obendrein enthält das vorangestellte Patronymikon auch noch die Synizese des $-\varepsilon \omega$ (die zwar einige wenige aber gleichfalls fragwürdige Parallelen hat) ${ }^{39}$. Und auch in diesem Fall darf nicht übersehen werden, dass diese Wendung $\Pi \eta \lambda \eta \bar{\imath} \alpha \delta \varepsilon \omega$ ' $A \chi \curlywedge \lambda \bar{\eta} \circ \varsigma$ in der Odyssee nur gerade in den beiden Nekyien wieder vorkommt (Od. XI 467, XXIV 15), sodass sie als bereits drittes markantes Element den Ilias-Introitus der Nekyia verbindet. Dazu kommt noch, dass die

35 So Il. VIII 379, XIII 831, XVII 241, XVIII 271, XXII 42, 335, 354, Od. III 259, XXIV 292.

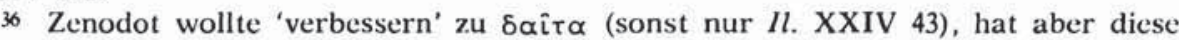
Dichtung dennoch nicht für echt gehalten und 4 f. athetiert. - Zum «mechanischen» Ubernehmen des Letzten Dichters ist von namhaften Forschern schon das Nötige gesagt, s. Peisistratos, S. 27 f.

37 So Wackernagel, «Idg. Dichtersprache», Philologus 95, 1942, S. 13 f. $\quad(=K l$. Schr., S. 198 f.). Er weist auch darauf hin, dass es sich um eine altidg. Erscheinung handelt, wahrnehmbar von den alten Indern bis zu den heutigen Russen. - Zwei scheinbare Ausnahmen von der Regel: a) Hugo Mühlestein weist den Verf. darauf

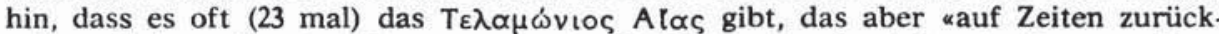
gehen dürfte, als $\tau \varepsilon \lambda \alpha \mu \omega v$ เo noch als Epitheton, noch nicht als Patronymikon verstanden wurde». b) die zweite s. gleich.

38 Auch das stellt Wackernagel ausdrücklich fest, a. O.

39 Zur Synizese s. Chantraine, Gramm. hom., I, SS. 69 f., 105 f., 200 f. (er sieht sie für spät an, obwohl ihm noch keine fertige Analyse zur Verfügung stand). Eine eigene Beobachtung dazu s. bald. 
gleiche Wendung in der Ilias noch an fünf Stellen begegnet, die ausschliesslich dem Letzten Dichter gehören ${ }^{40}$ : ein sehr eindeutiger Befund. Nimmt man auch hier wieder an, dass die Besonderheiten im Blick auf das Vorbild ihre Aufklärung erhalten, so wäre es das Bestreben des Letzten Dichters gewesen, den Orpheus-Vers von der Demeter Menis - der ihm bekannt gewesen sein müsste- um der Erhabenheit willen in bewusster Nachbildung zu adaptieren: d.h. nach dem

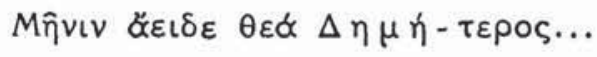

hätte er in vorsätzlicher Klang-Anapher das

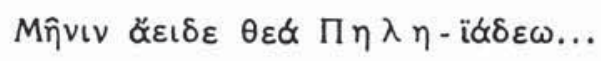

formuliert. Aus solcher Absicht würde sich beides - die Voranstellung des Patronymikon wie auch das In-Kauf-nehmen der Synizese- gut erklären ${ }^{41}$. Es läge bei diesem Sachverhalt abermals der Fall vor, dass man das Seltsame der späten unoriginalen Dichtung versteht, wenn man das Vorbild kennt, von dem der Letzte Dichter sich hätte inspirieren lassen. Dass dem auch die antike Auffassung entspricht - der Dichter

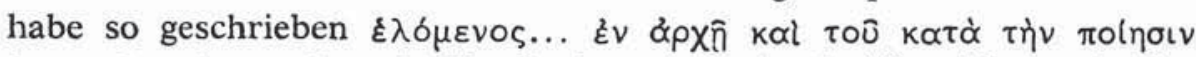
$\varepsilon^{2} \kappa \pi \varepsilon \sigma \varepsilon \hat{\imath} \nu \mu \varepsilon^{\prime} \tau \rho o u$ (womit die Synizese gemeint ist) ${ }^{42}$ - könnte in der Auffassung bestärken, dass auch hier die zu den zuvor angeführten Fällen analoge Erklärung berechtigt und richtig ist.

Jedenfalls: Wer synoptisch sieht, dass alle besprochenen Elemente des Introitus sonst nur beim Letzten Dichter begegnen $(\tau \grave{\alpha} \pi \rho \hat{\omega} \tau \alpha-$

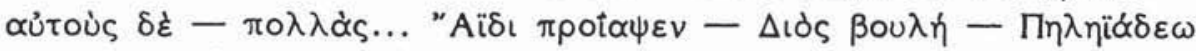

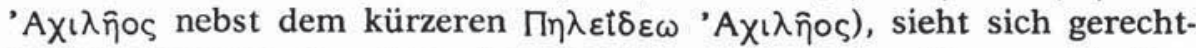
fertigt anzunehmen, dass der Eröffnungsvers von diesem epischen 'Redaktor' formuliert ist, und zwar auch hier als Sekundärdichtung nach einem ihm geläufigen Vorbild.

40 Die Stellen: Il. I 322, IX 166, XVI 269 und 653, XXIV 406 (s. VdM z. d. St.), wobei der Letzte Dichter ( $(\mathrm{B})$ ) ja auch der Nekyia-Dichter ist. Das kürzere $\Pi \eta \lambda \varepsilon t \delta \varepsilon \omega$

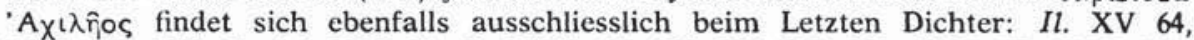
XVII 195, XX 85, Od. VIII 75. Il. XVII 105, 701, XX 312, 322, XXI 557, XXII 176. XXIII 542. s. VdM z. d. St.

41 Für Letzteres gibt es einen bemerkenswerten Parallelfall. In der alten Version

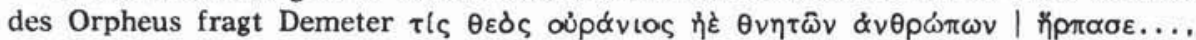
Als der Dichter des 'homerischen' Hymnus - unser Dichter- diese Frage der von ihm einbezogenen Hekate in den Mund legte, entstand sekundär $\tau$ (s $\theta \varepsilon \hat{\omega} \nu$ oủp $\alpha-$ $v(\omega v . .$. mit Synizese (H. Dem. 55, Peisistratos, S. 20 f.). Vgl. auch Richardson. S. 170 (unter Hinweis auf Hoekstra).

42 So Iustin a. O., s. Orph., fr. $48 \mathrm{~K}$. 
Oder sollte allein hier im ersten Vers ein einziges Mal das Umgekehrte vorliegen, dass die späte Einleitung - ungeachtet der dann

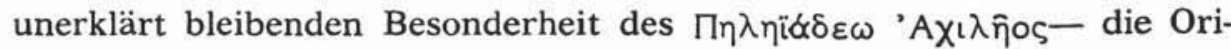
ginal-Dichtung darstellt, nach der der Orpheus-Vers von der Demeter Menis gebildet ist? Wenn nun aber die 'selbstverständliche' Priorität 'Homers' gegenüber 'Orpheus' ohnehin nicht mehr gegeben ist (oben S. 204), hat dieser 'Ausnahmefall' keinerlei Wahrscheinlichkeit mehr für sich. Wie in den andern besprochenen Fällen dürfte auch im Eröffnungsvers eher die Sekundärdichtung des Letzten Homerischen Dichters vorliegen.

Doch bevor man sich diese Erklärung wirklich zu eigen machen darf, müsste eine gewichtige Vorfrage geklärt sein: Kannte denn der Dichter das hier anzunehmende Vorbild, den Orpheus-Vers? Haben wir denn die Möglichkeit, ihm diese Kenntnis nachzuweisen? Was wissen wir in dieser Hinsicht über ihn?

\section{II}

Der entscheidenden Frage, ob der Dichter des Introitus (und der Nekyia), der Letzte Dichter am 'Homer', den Orpheusvers gekannt und im Aufblick $\mathrm{zu}$ ihm den Anfangsvers seiner Ilias geschaffen hat, gilt der zweite Teil unserer Betrachtung. Von diesem Dichter kann man inzwischen soviel wissen, dass es für eine klare Entscheidung in der noch offenen Frage des Il. I 1 ausreicht ${ }^{43}$. Das Wesentlichste ist hier zunächst in Kürze zu rekapitulieren: in einer solchen Kürze, dass es demjenigen Leser, der auf die ausführliche Darlegung nicht zurückgreifen will, als eine mögliche Hypothese dargeboten wird, die dann einfach nach ihren Konsequenzen beurteilt sein will.

Kontrovers und mithin ungelöst war lange das Problem des Verhältnisses der grösseren der unter Homers Namen überlieferten Hymnen $\mathrm{zu}$ den beiden Grossepen Ilias und Odyssee. Wer nun derjenigen Homeranalyse folgt, wie sie - nach dem Vorhergang von Eduard Schwartz und Erich Bethe- in Von der Mühll's Ilias-Hypomnema ihre vorläufige Vollendung gefunden hat, wird feststellen müssen, dass es zwischen Grossepen und Hymnen zwar zahlreiche dichterische Parallelen (wörtlich übereinstimmende poetische Elemente) gibt, die meist noch Träger besonderen mythischen Bildgehalts sind und jeweils gleichen mythischen Assoziationen angehören, dass es sich dabei jedoch

43 Eigens über den Letzten Dichter handelt Verf. in Peisistratos und sein Homerischer Dichter. Ein Kapitel Prolegomena ad Homerum, mit 3 Abb., Bern und München 1983. 
ausschliesslich um Parallelen zwischen den Hymnen und solchen Partien in Ilias und Odyssee handelt, die - nach der Analyse- dem Letzten Dichter ( $\mathrm{B} »)$ gehören. Die Folgerung aus diesem Befund ist unvermeidlich: Da es undenkbar ist, dass ein späterer Dichter bei Schaffung der Hymnen rein zufällig nur gerade solche 'Vorbildstellen' im 'Homer' berücksichtigt und benutzt hat, und undenkbar auch, dass er selbst Homeranalyse getrieben oder diese berücksichtigt habe, wird man anzunehmen genötigt, dass der Schöpfer der Hymnen mit dem Letzten Dichter der Grossepen identisch ist, sodass es sich in den Parallelen um mehrfache Verwendung ihm geläufiger poetischer Wendungen durch diesen Dichter handelt ${ }^{44}$. Für diese Auffassung spricht auch, dass oft ein Variantenspiel zu beobachten ist, das keine einseitige Abhängigkeit anzunehmen erlaubt ${ }^{45}$. Und durch eine verräterische Einzelheit sieht man sich schliesslich vollends gerechtfertigt. Der Letzte Dichter kennt nämlich - wie die Ilias vielfach zeigt- Kleinasien von Nord nach Süd sehr genau, hat sich dort also länger oder öfter aufgehalten: und in einem Hymnus gebraucht er einmal unversehens ein thrako-phrygisches Wort, das im 6. Jahrhundert nur in Kleinasien in Gebrauch war ${ }^{46}$.

Nun ist zwar zuzugeben, dass diese Identität des Letzten Dichters mit dem Hymnendichter mit Sicherheit zunächst nur für den Demeter= und den Aphroditehymnus gilt ${ }^{47}$. Doch das genügt für die entscheidende Argumentation vollauf. Denn der Demeterhymnus lässt über seinen Dichter nicht den geringsten Zweifel. Es ist da ein doppelter Sachverhalt $\mathrm{zu}$ beobachten, dem man nicht ausweichen kann:

A. Der 'homerische' Demeterhymnus ist als Kleinepos homerischer Erzählart auf der Grundlage des älteren 'Hymnus' des Orpheus geschaffen: der unvoreingenommene kritische Vergleich aller parallelen Passagen zwischen 'Homer' und 'Orpheus' (fr. 49) - sprachlich wie inhaltlich- lässt unerbittlich keine andere Schlussfolgerung $\mathrm{zu}^{48}$.

B. Der Dichter des 'homerischen' Hymnus hat durch eine längst erkannte Erweiterung die Göttin Hekate - und zwar die 'Ekớn $\sigma \varepsilon \varepsilon_{\lambda} \alpha \varsigma$

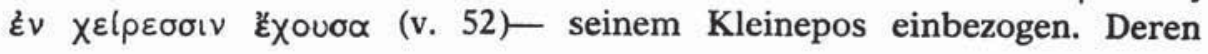

44 Diese (rund ein halbes Hundert) Parallelen mit ihren Besonderheiten sind im Peisistratos besprochen, Abschnitt 3 (SS. 41-60).

45 Paradigmatisch z. B. Peisistratos, S. $53 \mathrm{ff}$.

46 Peisistratos, SS. 38-40, 60. Hierher gehört auch, dass er den Sänger Phemios,

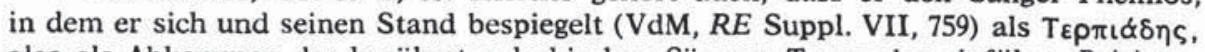
also als Abkommen des berühmten lesbischen Sängers Terpander einführt, Peisistratos, S. 41.

47 Für den Aphrodite-Hymnus nahm schon VdM diese Identität an (zumal auf Grund der Aneadenprophezeiung): Hyp., SS. 76, 223, 303 ff. (bes. Anm. 33), 325, 51; Peisistratos, S. 61 .

48 S. oben Anm. 5. 
Rolle wird "stark hervorgehoben, ist aber sozusagen an den Haaren herbeigezogen... Der Wunsch des betreffenden Dichters, Hekate einzuführen, war aber so gross, dass er auf jegliche Motivation verzichtet» ${ }^{49}$. Diese Göttin, die Hekate "selasphoros», gibt es aber nur im Gentilkult der Lykomiden im attischen Phlya, weshalb der Dichter, dem sie so wichtig ist, dass er sie geradezu gewaltsam dem Hymnus einbezieht, nur ein Lykomide gewesen sein kann ${ }^{50}$.

Sind nun diese beiden Sachverhalte als Prämissen richtig, so befindet man sich im Zwange eines Syllogismus: der ältere Hymnus des 'Orpheus' als Grundlage des 'homerischen' Kleinepos muss also dem Ly komiden bekannt gewesen sein. Und das lässt sich nun in der Tat als zutreffend verifizieren: es ist nämlich sowohl bezeugt als auch nachweisbar, dass die musisch-poetische (d.h. die religiöse) Gentiltradition der Lykomiden seit den Zeiten des Neleischen Pylos eine Orpheustradition war ${ }^{51}$.

Ist also der Dichter des 'homerischen' Demeterhymnus als Lykomide gesichert, und ist er mit demjenigen identisch, der als Letzter Dichter zur Peisistratidenzeit die homerischen Dichtungen zu den Grossepen (dem 'attischen Homer') zusammengearbeitet hat (also mit dem Dichter "B» der Analyse) ${ }^{52}$, so ist sofort alles klar: aus der ihm als Gentilerbe vertrauten Orpheustradition hat er sich in seinem homerischen Dichten anregen lassen, und das nicht nur zur Schaffung des 'homerischen' Kleinepos von Demeter, sondern ebenso auch zu seinem feierlich, im Hymnenstil, anhebenden Introitus zur Ilias. Als Lykomide kannte er die Orpheusdichtung von der Demeter Menis nicht nur, sie war ihm erhabenes Vermächtnis, zu dem er aufblickte: und aus der Nachbildung

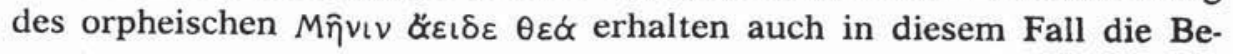
sonderheiten und Seltsamkeiten ihre Aufklärung.

49 Dies Urteil bei M. P. Nilsson, Gesch. gr. Rel. I 3, S. 723.

50 So Peisistratos, SS. 20-22, wo nur die Ausdrucksweise (S. 22, Z1. 6-15) für einen archäologischen Leser missverständlich sein könnte: denn nicht die 'Fackeln' sind das Besondere der Göttin - solche hat Hekate so gut wie immer und überall-

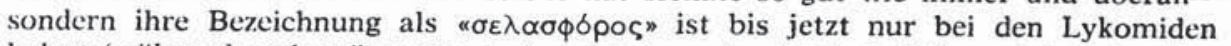

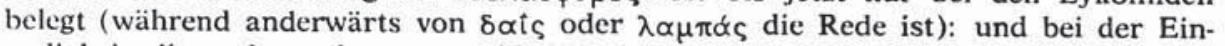
maligkeit dieses besonderen sprachlichen Ausdrucks kann es nicht blosser blinder Zufall sein, dass der Dichter just diese den Lykomiden eigene Sprachform gebraucht.

51 Die Bezeugung: Hippol., Refut. V 20, 5 f. (III 121 f. Wendland). Pausan., I 31, 4. IV 1, 5. 1, 7 f. X 30, 12 (vgl. I 22, 7 und IV 1, 5). Dazu Peisistratos, SS. 22 24. - Der Nachweis: Peisistratos, Ss. 61-75.

52 VdM nennt ihn oft den aIliasdichter» (so z. B. Hyp., SS. 23, 31, 55 usw.). Es ist also derjenige, der hier wie dort im Interesse Athens (und der peisistratischen Politik) Erweiterungen eingefügt hat, z. B. die Demos-Eponyme Melite im Demeterhymnus (Peisistratos, SS. 24-27) und die berühmt-berüchtigte Athenerpartie in der Boiotia seiner Ilias.

LIV, $20^{\circ}-3$ 
Dies jetzt schon sich abzeichnende Ergebnis könnte manchem als zu kühn erscheinen (zumal wem es schwer fällt von der alten These der 'orphischen Plagiatliteratur aus Homer und Hesiod' Abschied zu nehmen). Und selbst wer der Logik unserer Schlüsse zu folgen vermag, wird doch wohl eine kritische Frage stellen, nämlich diese: Wohl stünde der Eröffnungsvers innerhalb der Ilias-Einleitung als Sekundärdichtung nicht allein -einige andere Fälle innerhalb der nur sieben oder acht Verse umfassenden Proekthesis sind ja besprochen-, aber es wäre doch zu fragen, ob die Schlussfolgerung - wonach der Lykomide aus der ihm als Gentilerbe vertrauten Orpheustradition sich in seinem ho. merischen Dichten hat anregen lassen- auch verifizierbar ist, d.h. konkret: ob der Fall des Il. I 1 als Orphe us-Nachahmung in den Grossepen singulär bleibt, oder ob etwa noch andere Orpheus-Reminiszenzen in seinem 'attischen Homer' nachweisbar sind, sodass die gegebene Erklärung von daher auch als genügend gesichert und glaubwürdig angesehen werden kann. War dem Letzten Dichter das lykomidische Gentilerbe, alte musische Orpheustradition, vertraut, so müsste er doch wohl mehr als nur ein einziges Mal im Aufblick zu jener altgeheiligten Muse gedichtet haben, auf Einen Fall könnte etwas derartiges dann wohl kaum beschränkt sein. Und auch uns müsste einiges davon noch fassbar sein.

In der Tat: Il. I 1 steht beim Letzten Dichter mit seiner Provenienz aus alter Orpheustradition nicht allein. Darf man beim Rückgriff auf praeliterarische epische Tradition die Erwartungen in der Nachweisbarkeit nach Lage der Dinge nicht zu hoch schrauben, so können doch einige im vorliegenden Zusammenhang relevante - und sogar recht bemerkenswerte- Fälle das Erwartete zeigen: dass nämlich der lykomidische Dichter in seinem 'Homer' nicht allein den Menis-Vers seinem musischen Gentilerbe verdankt.

Gleich in der dem Ilias-Introitus so eng verbundenen Nekyia - die ja mitsamt der Kirke-Dichtung ganz dieses Dichters Schöpfung istfindet sich der misogyne Vers

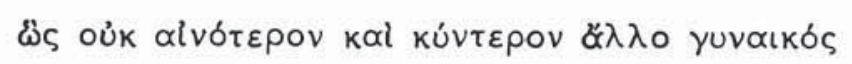

(Od. XI 427). Er entstammt der Orpheustradition, geht in seiner ältesten Form bis auf den Sänger zurück, dessen 'Misogynismus' jetzt als Ausdruck seiner antimatriarchalen historischen Position erkennbar ist ${ }^{53}$. Der Vers war überaus berühmt, weshalb er auch im 'orphischen' Schrifttum noch seinen Platz behielt (fr. 234). Auf das Einzelne muss hier

53 Sänger d. Vz., SS. 26-29, 39-52. Vgl. Peisistratos, S $104(« 4 n)$. 
nicht mehr eingegangen werden, nachdem über ihn schon mehrfach gehandelt ist ${ }^{54}$. Der Letzte Dichter macht jedenfalls von diesem famosen orpheischen Erbstück mehr als nur einmal Gebrauch, und zwar auch in nichtmisogynen Assoziationen (also unter sekundärer Verwendung der poetischen Fügung, wie das auch an andern Beispielen noch zu beobachten sein wird): ausser in der Nekyia auch in seinem VIII der Ilias (423 und 483), sodann in übertragener Benutzung des Uberkommenen an zwei weiteren Stellen der Odyssee (VII 216 und XX 18) ${ }^{55}$, sowie zweimal in seinem Demeterhymnus (90 und $305 \mathrm{f}$.). Und an allen diesen Stellen liegt die alte Schelte in der gle i chen jungen Umformung vor d.h. in der dem Lykomiden eigenen $U m=$ oder Neuformulierung.

Andere bemerkenswerte Fälle von Rückgriff auf das Orpheus-Erbe führen thematisch näher an $\mathrm{Il}$. I 1 heran. Denn den mythisch-religiösen Kern der lykomidischen Gentiltradition bildet jene der Feier ihrer Hauptgottheiten zugeordnete hymnische Dichtung von Verschwinden und Wiederkehr der Göttin und vom $\mathrm{Z}$ orn der Demeter, also jenes musisch-poetische Gut, das auch in historischer Zeit noch als 'die' Orpheus-Dichtung schlechthin galt ${ }^{56}$. Ihm entnimmt der Letzte Dichter mehrfach einzelne geläufige Elemente.

In der alten Orpheus-Tradition waren Verschwinden und Wiederkehr der Göttin rein mythisch lokalisiert. Geraubt worden sei sie $\varepsilon_{\kappa} \tau \hat{\omega} \vee \pi \varepsilon \rho t$ $\tau o ̀ v ~ ' \Omega \kappa \varepsilon \alpha \nu o ̀ v ~ \tau o ́ ~ \pi \omega \nu$, also vom mythischen 'Leimon' am Rande des Okeanos, und zwar aus dem Kreise ihrer Gespielinnen, die ja Töchter des Okeanos waren ${ }^{57}$. Entführung und Wiederbringung, Kathodos und Anodos, das 'Hinüber' und 'Herüber' vollzog sich demnach an der altorpheischen ('schamanistischen') Weltenscheide zwischen Dies= und Jenseits, Tag = und Dunkelwelt: also 'da', wo Grenze und Ubergang der beiden kosmischen Halbheiten sind, am Strome des Okeanos wo sich der Leimon, 'da' wo auch das Tor und die eherne Schwelle sich befinden, wo der Gott mit dem Stabe waltet, also

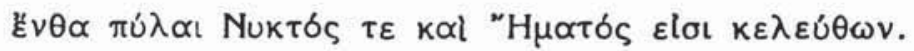

54 Orpheus, S. 33 f., Sänger d. Vz., S. 18 f., 26-29, Peisistratos, S. 32, 45 f.

55 Beide Stellen gehören "B» nach VdM ad $l l$.

56 Nach dem Marmor Parium (Kern test. 221), s. oben Anm. 6.

57 Orph., fr. 43 und 49, 19 ff. Dass alle diese Elemente -Okeanos, Leimon, Töchter des Okeanos- zusammenstimmen ist offenkundig. Zum Leimon: Il. XVI 151. Orph., fr. 222, 3. Orph. Hy. 18, 13 und 29, 12 (auch 43, 3 und 51, 4). 'Hom.' H. Dem. 7 und 417 (wo der Okeanidenkatalog freilich durch einige Nicht-Okeaniden -im Interesse Athens- erweitert ist). - Orpheus, S. 227 f., Peisistratos, SS. 24-26. 
Die heilige Geschichte der Göttin als ein Geschehen in dieser mythischen Assoziation ist ja auf dem Grossen Goldring von Mykenä dargestellt: sodass das altorpheische poetische Element von der Begegnung der Wege der Nacht und des Tags - das schon sprachlich als uralt ausgewiesen ist- zweifellos Hauptbestandteil dieser Assoziation bildet: also einer mythischen d.h. religiösen Assoziation ${ }^{58}$. Aus diesem Ursprung ist noch in früher historischer Zeit die "Begegnung der Wege der Nacht und des Tags» den der Tradition folgenden Dichtern - Hesiod, Pindar und Parmenides sind zu nennen- immer noch unverändert jenes mythische Element, das Grenze und Ubergang zur J en seit s welt bezeichnet ${ }^{59}$. Auch der Lykomide kennt diesen altertümlichen Orpheus-Vers, den er in seiner Kirke-Nekyia-Dichtung verwendet - jedoch vollkommen unmythisch und äusserst banal: Odysseus gelangte nach Telepylos, der Lästrygonenstadt, wo der Hirt den Hirten grüsst, wenn der eine hinein = der andere heraustreibt: ein schlafloser Mann könnte da doppelten Lohn verdienen, nämlich als Rinder $=$ wie auch als Schafhirt, weil die Bahnen von Nacht und Tag sich da so nahe sind:

$$
\begin{aligned}
& \ddot{\delta} \theta \iota \pi
\end{aligned}
$$

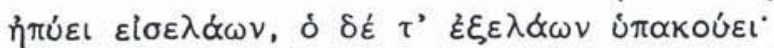

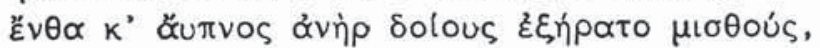

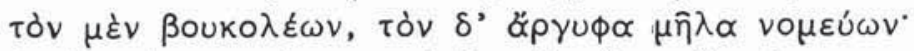

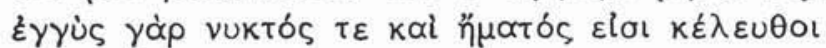

(Od. X 82-86). Ein altehrwürdiges mythisch-poetisches Traditionselement, das den andern Dichtern immer noch der Jenseitsassoziation angehört, ist hier frivol zu einer phantastischen Charakteristik einer AbenteuerStation verwendet -so unmythisch und irreligiös, dass spätere antike wie auch moderne Interpreten beim Lesen dieses Passus geographisch an den hohen Norden denken ${ }^{60}-$ : es ist prof a niert und als poetische Formel zum Vehikel eines absonderlichen Gedankens gemacht. Und das kennzeichnet den Letzten Dichter: er ist im Grunde irreligiös und vermarktet unbedenklich sein Gentilerbe d.h. das Gut der ihm tradierten altorpheischen Muse ${ }^{61}$.

58 Orpheus, S. 253 f. Uber den Vers selbst ist mehrfach gehandelt: Orpheus, SS. 35-37, Sänger d. Vz., SS. 10-31 (hier auch das linguistische Argument der auf

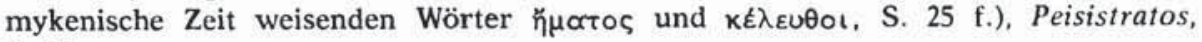
S. 31 .

59 Hes., Th. 748 ff., 811, Parm., fr. 1, 11 f., Pind., Pyth. IV 195 ff.

60 Krates von Mallos: schol. HQ ad Od. X 86. Ameis-Hentze, Od. erkl. (1922) ad l., Cauer 3 , S. 641, RE Suppl. V 538, 54 ff.

61 Peisistratos, S. 78 f., wo auch auf VdM's entsprechendes Urteil verwiesen ist, S. $79,2$. 
Diese Art des Umgangs mit dem Ererbten lässt sich bei ihm mehrfach beobachten. Das mag hier zunächst an einigen Elementen seiner Dichtung vom Phäakenpalast gezeigt werden.

Die mit Bestimmtheit dem Letzten Dichter zugewiesene Partie, Od. VII 78-132 62, beginnt sehr bezeichnend damit, dass Athene, nachdem sie den Odysseus über die Phäaken aufgeklärt und ihm guten Rat erteilt hat, Scherie verlässt um nach $\mathrm{Mar}$ a thon und Athen zu gelangen, wo sie sich ins Haus des E re cht he u s begibt: das ist geradezu ein Siegel des attischen Dichters, des Lykomiden, des Schöpfers des 'attischen Homer'. Odysseus aber geht zum Palast des Alkinoos, macht sich dabei einige Gedanken, bevor er an dessen Schwelle kommt,

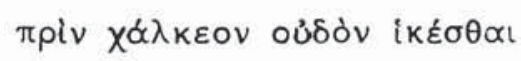

(Od. VII 83). Nun ist die "eherne Schwelle» seit alters ein Element der gerade besprochenen -orpheischen- Jenseitsassoziation. Und so kennt sie ja auch Hesiod: als die grosse Schwelle zur Dunkelwelt, zum Reiche der Kinder der Nacht, Schlaf und Tod, als Eingang zur Unterwelt (Th. $736 \mathrm{ff}$.), und es ist diese Schwelle, bei deren Uberschreitung sich Nacht und Tag begegnen,

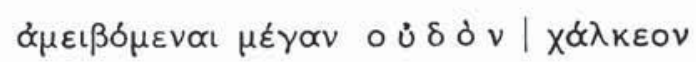

(Th. 749 f.). Auch in der zweiten (parallelen) Version gehört diese Schwelle der gleichen Assoziation an: wo die Quellen und Wurzeln aller Dinge sind und die Jenseitswelt sich auftut, beim Okeanos, findet sie sich:

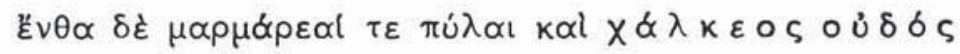

(Th. 811). Dies Traditionselement der orpheischen Jenseitsassoziation hat natürlich auch der Lykomide gekannt und verwendet:

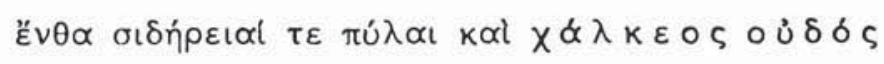

(Il. VIII 15). Die mythische Assoziation ist zudem auch daran kenntlich,

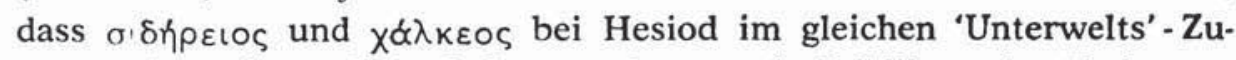
sammenhang ihren Platz haben, und zwar als Prädikate des Todes:

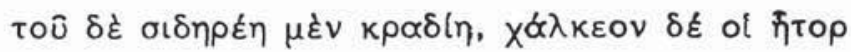

$62 \mathrm{VdM}, R E$ Suppl. VII 715, 6-52. Entscheidend auch Bethe $\mathrm{II}_{2}, \mathrm{~S} .336 \mathrm{ff}$. 
(Th. 764). Ebenso gehört auch für Parmenides die "Schwelle» zum Tor, da sich die Wege der Nacht und des Tags begegnen:

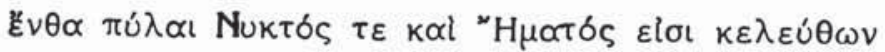

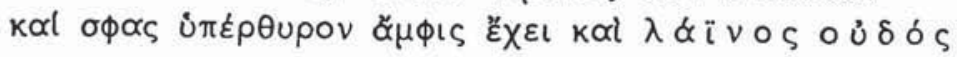

(fr. 1, 11 f.). Die hier "steinerne" Schwelle ${ }^{63}$ ist aus dem alten orpheischen Ursprung unverändert immer noch Schwelle zum 'Jenseits', und zwar in einem älteren und ursprünglicheren Sinne als bei Hesiod, insofern dies 'Jenseits' dem Eleaten -im Gegensatz zum Böoter- noch nicht 'Unterwelt' ist ${ }^{64}$. Diese Diskrepanz (als Erstes) sowie auch der Wechsel $\lambda$ d́́ivos gegenüber $\chi \alpha \dot{\lambda} \kappa \varepsilon \circ \varsigma$ (als Zweites) zeigen das Gleiche wie (als Drittes) die in sich allein schon auffälligste Diskrepanz, die infolge Hesiods jüngerer Umformulierung der Begegnung der Wege der Nacht und des Tags entstanden ist ${ }^{65}$ : dass Parmenides offenkundig nicht dem Böoter sondern unmittelbar der älteren Orpheustradition folgt, die ihrerseits ja auch der hesiodeischen 'Unterwelts'-Dichtung zu Grunde liegt ${ }^{\text {to }}$. Von allem Anfang an ist also die Einbindung der Grossen Schwelle in diese Jenseitsmythologie unverkennbar: sie ist ein mythisch-poetisches Element, das Grenze und Ubergang zur Jenseitswelt bezeichnet.

Aus dem Gentilerbe der Lykomiden ist sie als Traditionsgut der orpheischen Muse auch dem Letzten Dichter geläufig. In seiner Dichtung vom Alkinoospalast hat er die poetische Fügung benutzt, und zwar als ein ihm wohlvertrautes Element gleich zweimal (Od. VII 83 und 89): aber ebenso abgelöst von der mythischen Jenseits-Assoziation und als Schwelle eines Palastportals ebenso profaniert wie den altorpheischen Vers von den Wegen der Nacht und des Tags in der fabulosen Schilderung der Lästrygonenstadt. Beide Fälle zeigen, welcher Art die Quelle ist, der er seine homerisch fabulierenden Eingebungen verdankt, und beide zeigen auch die bedenkenlose Profanation der altgeheiligten

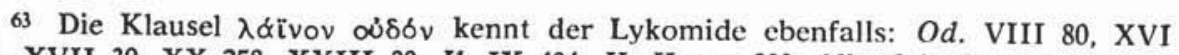
41, XVII 30, XX 258, XXIII 88, Il. IX 404, H. Herm. 233. Alle Od. - Stellen dürften ihm gehören. Zu Od. XVI $40 \mathrm{f}$. ist VdM's Bemerkung «könnte 40 unmittelbar anschliessen" inhaltlich gemeint, dichterisch formuliert sind $40 \mathrm{f}$. doch wohl noch von "B». Zu Od. XVII 30 folgt man anstatt Kirchhoff besser Schwartz.

64 Neuplatonisch-christliche Begriffe wie 'Himmel'= oder 'Höllen'-fahrt, 'hinauf' oder 'hinab' lehnt z. B. auch K. Deichgräber für Parmenides mit Recht ab: Phronesis 14,1969, S. 15.

65 Parm., fr. 1, 11 f. Hes., Th. 748 ff.: Parmenides hat hierin auch die gegenüber Hesiod ältere poetische Form.

66 Sänger d. Vz., SS. 12-26. Peisistratos, S. 31. Weiteres in Die Verkannte Muse, Bern 1986, bes. SS. 93-103. 
Muse, ihre Benutzung als Steinbruch für die Aufführung ganz andersartiger Gebäude.

Kurz darauf folgt in dieser Palast-Dichtung ein weiteres seinem Ursprung entfremdetes Traditionselement. Schon in der vorhomerischen - unter den Namen des Orpheus und Musaios überlieferten- Form des Demeterhymnus gehört es zu den Segnungen der Göttin, dass sie die von Alter und Tod bedrohten Menschen unsterblich machen könne. So hätte sie auch das ihr von Baubo bezw. Metaneira übergebene Kind unsterblich gemacht:

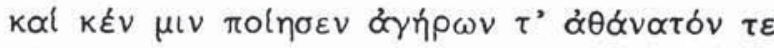

$$
\begin{aligned}
& \varepsilon l \mu \grave{\eta} \ldots \text {, }
\end{aligned}
$$

nämlich wenn sie dabei nicht beobachtet worden wäre ${ }^{67}$. Und in ihrer Scheltrede auf die unseligen Menschen spricht sie es nochmals aus:

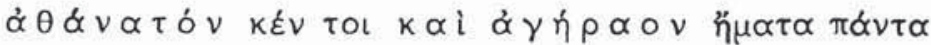

$$
\begin{aligned}
& \pi \alpha \hat{i} \delta \alpha \phi i \lambda \circ v \pi \circ i \eta \emptyset \alpha
\end{aligned}
$$

(H. Dem. 260 f.). Auch dieser Vers von Tod= und Alterslosigkeit ist ein sehr altes musisches Element: er begegnet als eine dem Hesiod bereits überkommene poetische Prägung in dessen Theogonie:

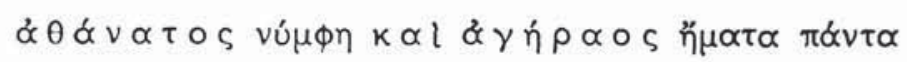

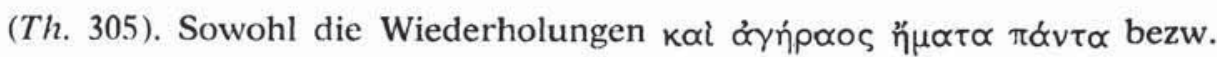

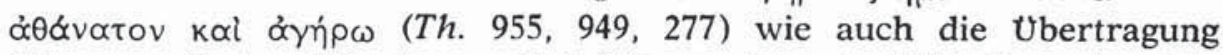
dieser poetischen Wendung auf Echidna zeigen deutlich, dass sie nicht hesiodeische Schöpfung sondern ihm bereits vorgegebenes poetisches Gut ist: beim Orpheus-'Hypoklepten' Hesiod - und zumal als demetrisches Element- natürlich aus der alten Orpheustradition. Und als altes Element dieser, der orpheischen Muse, ist der Vers schliesslich auch durch seine Bewahrung in den 'orphischen' Literaturpoesien ausgewiesen (Orph. fr. 228c) ${ }^{68}$. (Auf dieser uralten mythischen Vorstellung von

67 Orph., fr. 49, 81-101, 'Hom.' H. Dem. 219 ff., bes. 242 f. In der Orpheustradition hatte das Entsprechende schon scinen Platz, ein \&yń $\left.\rho^{\prime} \alpha \circ v\right)$ ist noch erhalten, der Text aber nicht mehr restituierbar, Versuche bei Kern p. 124. Die 'Orpheus'-Version ist gerade auch in diesem Motiv ersichtlich älter als die 'homerische': s. Murray (oben Anm. 5), dem auch Richardson folgt, ad 254. Und sie war auch im Altertum mindestens ebenso bekannt wie die 'homerische': das zeigt Apd., I 5, 1, wozu

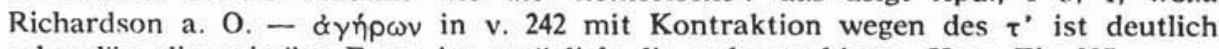
sekundär, die primäre Form ist natürlich die unkontrahierte, Hes., Th. 305.

68 Mit dem ursprünglich zugehörigen $\eta_{\mu \alpha} \alpha \alpha \pi \alpha v \tau \alpha$ ist auch sein hohes Alter ausgewiesen, s. Orpheus, S. 206. - Seine Zugehörigkeit zur Orpheustradition: Peisistratos, S. 44 f. 
Tod $=$ und Alterslosigkeit beruht ja auch die Eos-Tithonos-Geschichte). Dem Lykomiden ist auch dieser Vers aus seinem Gentilerbe geläufig, wie die in seinen Partien festzustellende öftere Verwendung zeigt, allem voran natürlich im Il. VIII und im Aphroditehymnus ${ }^{\oplus}$. In seiner Diehtung vom Phäakenpalast fährt er in der Schilderung jenes Portals mit der «ehernen Schwelle» so fort, dass zu dessen beiden Seiten goldene und silberne Hunde angebracht waren, die Hephaistos eigens kunstvoll gefertigt hatte,

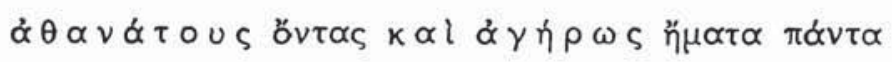

(Od. VII 94): Kunstgebilden aus Edelmetall kommt hier die Tod $=$ und Alterslosigkeit zu, doch wohl weil Silber und Gold weniger der Korrosion ausgesetzt sind als andere Werkstoffe, oder weil Hunde aus solch edlem Material entschieden langlebiger sind als natürliche Hunde. So oder so: banaler geht's nicht mehr. Wiederum ist hier ein Element der ihm tradierten orpheischen Muse profaniert - übel profaniert (darf man ruhig sagen)- und ganz aus der Assoziation gelöst, der es entstammt. Unnötig auf sein attisches $\iota v \tau \propto \varsigma$ hinzuweisen, nachdem er seiner Dichtung mit der Hervorkehrung von Marathon, Athen und des Hauses des Erechtheus ohnehin schon seinen Stempel aufgedrückt hat.

Aber es kommt bald noch besser. Wenn etwas im Demetermythus uralt ist und unlösbar zu der ihre geraubte Tochter suchenden Göttin gehört, so sind es die Fackeln, die sie in Händen hält. Das ist in der gesamten griechischen Welt genügend bezeugt ${ }^{\text {to }}$ und gehört zu Kulten, die jeder Art fassbarer Dichtung an Alter überlegen sind. Kultbilder waren daher oft mit einer oder zwei Fackeln ausgestattet, so z.B. in einem Heiligtum der Demeter Erinys im Gebiet der Stadt Thelpousa in Arkadien oder namentlich auch im Despoinaheiligtum zu Akakesion, wo die Göttin eine Fackel in ihrer Rechten hält ${ }^{71}$. Daher begegnet die mit Fackeln in den Händen suchende Demeter auch im Hymnus zwei-

69 It. VIII 539, H. Aphr. 214. Ferner: Od. V 136, VII 257, XXIII 336, dazu auch mit leichter Variante oder teilhaft: Il. XII 323, XVII 444, II 447, Od. V 218, H. Aphr. 221 und 240, H. Ap. 151 (Zu Od. V 136: 125-28 und 133-36 dürften Erweiterung von $* \mathrm{~B} *$ sein, 134 init. $=130$ init., 134 etwas inkonzinn zu 130, dazu das Einsetzen wie Od. I 11. Zu Od. V 218 s. Düntzer ad 201-27, Ameis-Hentze zu 192-227 und Krit. Anhg. ad 204 und 205).

70 Demeter als Fackelträgerin begegnet sogar auf Münzen, s. Roschers Lex. II 1, 1288, 50 ff., 1346, 14 ff. Zu den Fackeln in Demeterkulten s. Richardson, S. 165 und $167 \mathrm{f}$.

71 Pausan. VIII 25, 4-7 und 37, 3 f. «Fackeln» (plur.) in Stiris in Phokis, Pausan., X 35, 10, in Henna, Cic., in Verr. IV 109. Vgl. auch Roschers Lex. II 1, 1351, 36 ff., 1353, 1 ff. (Bei Vorliegen des LIMC «Demeter» wird noch mehr zu Tage kommen). 
mal, wo gerade dies Element fraglos übernommen und nicht erst Neue-

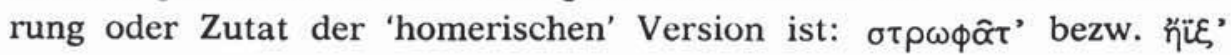

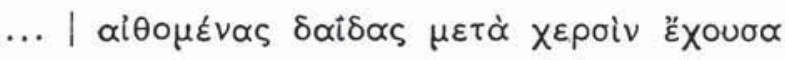

(v. 48 und 61). Als alt erweist sich dies poetische Element schon dadurch, dass es in gleicher Position auch als hesiodeisch begegnet:

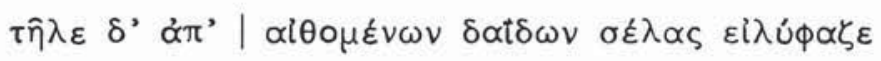

(Sc. 275), und uralt ist der Vers ja auch in seiner sprachlichen Bildung: das " $\alpha i \theta \rho \mu \varepsilon v \propto \varsigma$ " ist ein -in historischer Zeit erstarrt und in nur noch drei Wendungen formelhaft vorkommendes- Relikt eines Verbum, das in 'achäischer' Zeit in Gebrauch war ${ }^{72}$. Jener Welt entstammt also die poetische Prägung, die ja — sowohl nach der Konkordanzenregel wie auch als Primärbestandteil des Demetermythus- Element der Orpheustradition war ${ }^{73}$. Also auch dies ein dem Lykomiden aus seinem Gentilerbe vertrautes Element, das er in seiner Dichtung vom Alkinoospalast verwendet: und zwar um dessen Beleuchtung zu schildern - dass nämlich auf schönen Sockeln goldene Jünglinge stehen mit den Fackeln in den Händen, um nächtliche Gelage zu erhellen:

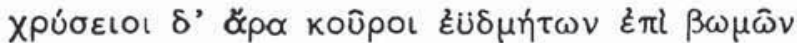

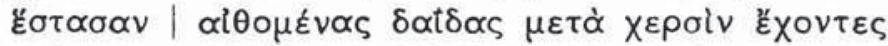

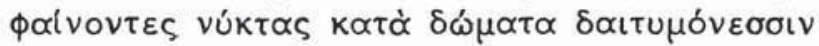

$(\mathrm{Od}$. VII $100 \mathrm{ff}$.). Das ist abermals die bedenkenlos profanierende Benutzung der altgeheiligten Muse: aus mythischer - einem Priestergeschlecht bis dahin mündlich überkommener- Sakraldichtung, aus der Dichtung des Orpheus um Demeter, wird so säkularisiertes 'homerisches' Fabulieren.

Auch der an den Fackelvers unmittelbar angeschlossene (v. 102) dürfte ein weiteres Mal das Gleiche darstellen. Denn dieser ist doch wohl nur Variante der altberühmten noch in historischer Zeit geläufigen orpheischen Prägung von den Wegen der Nacht und des Tags, die der Letzte Dichter ja auch in seiner Lästrygonenpartie zu profaner Phantastik benutzt hat. Das wird sofort erkennbar, wenn man nur einmal die Modifikations $=$ und Variationsformen dieser Fügung zu einer Reihe vereinigt:

72 C. J. Ruijgh, L'élément achéen dans la langue épique, Assen 1963, S. 130 f. Orpheus, S. 207 f. mit den Anm. 4, 5 und 1.

73 Orpheus, S. 207 f. Peisistratos, S. 52. 


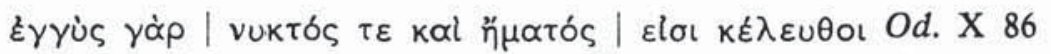

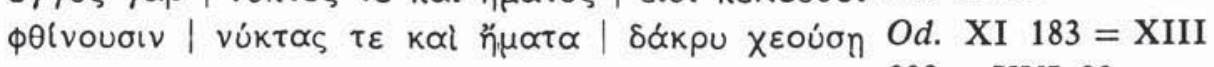

$338=$ XVI 39

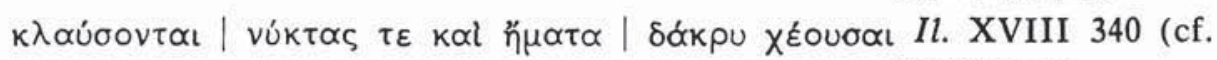
XXIV 745)

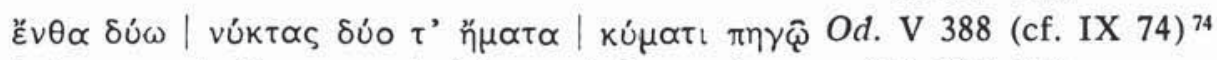

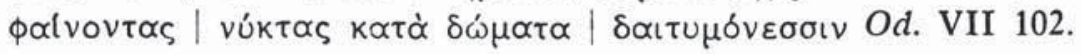

In der den Lykomiden als Gentilerbe vertrauten orpheischen Muse war der Letzte Dichter ja durchaus zur Eigengestaltung nach Art der 'Oral poetry' fähig: d.h. zum Variieren, Neuformulieren und freien Kombinieren ${ }^{75}$. Das schliessliche Ergebnis solchen Umgestaltens ist dann ein Vers, dem man die mythisch-religiöse Provenienz nicht mehr ansieht: ein profaner Erzählvers, den jeder für 'echten Homer' nimmt ${ }^{76}$. Und das dürfte auf weitere Teile des uns überlieferten Epos zutreffen: dass Orpheus letztlich dahinter steht wird man nur in seltenen Fällen noch wahrnehmen können.

Die Dichtung vom Alkinoospalast ist jedenfalls für die Schaffensweise des Letzten 'homerischen' Dichters im Attika der Peisistratidenzeit paradigmatisch. Sooft es geht macht er Gebrauch von der ihm aus Familientradition zu Gebote stehenden musischen Orpheustradition: wobei er jedoch nur die geprägten Formen benutzt, um sie in neuem Geiste neuartigen Zusammenhängen dienstbar zu machen ${ }^{n}$. Das führt zu einer oft bedenklichen Profanation des Tradierten. Solche Verfremdung der altgeheiligten Muse war in der Ubergangszeit des 7. und 6. Jahrhunderts nicht so unerhört: schon Hesiod hat einen orpheischen Unterweltsvers auf die Neger umgedeutet, und auch beim berühmten Terpander darf man wohl an Ähnliches denken, wenn überliefert war, dass er in seiner orpheisch-lesbischen Kitharodie auch 'Homer' nachgeahmt habe ${ }^{78}$.

Auch aus der Ilias sind jetzt noch einige Beispiele anzufügen, die von paradigmatischer Bedeutung sind und $\mathrm{zu}$ dem sub judice stehenden

\footnotetext{
74 Das $v$-Ephelkystikon zur Schaffung einer Länge (Od. XI 183 usw.) ist Sekun-

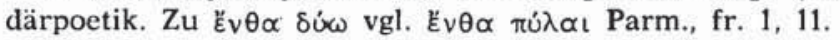

75 Peisistratos, SS. $27-29$ und 37 f., dazu auch 43, 46, usw., 59.

76 Dazu Peisistratos, S. 79 Mitte.

77 So also zunächst in $83,89,94,101,102$. Kleinere poetische Elemente dieser

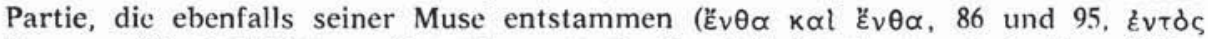
ह̌epyov, 88) sind hier zu übergehen, da sie ja nicht in ebenso eindeutiger Weise zur Kennzeichnung seiner Dichtmanier beitragen.

78 Zu Hesiod: Orpheus, S. 42 ff., Sänger $d$. Vz., S. 74 f., Peisistratos, S. 33 f. Zu Terpander: Sänger $d$. Vz., S. 18, 1, 67, Peisistratos, SS. 41, 69 f., 94.
} 
Il. I 1 hinführen werden: also poetisches Gut der orpheischen Muse aus dem Bereich der Demeterdichtung ${ }^{77}$.

Zwei der bereits besprochenen poetischen Wendungen, nämlich den misogynen Orpheusvers sowie den von Tod $=$ und Alterslosigkeit hat der Letzte Dichter ja auch in seinem VIII der Ilias verwendet (in den vv. 423, 483, 539). Und eben diese seine VIII-Dichtung enthält noch mehr Traditionsgut der orpheischen Muse. So zunächst in den Versen 19 und 20 sowie 181 , von denen hier abzusehen ist, da sie nicht dem Bereich des Demetrischen entstammen ${ }^{80}$. Sodann aber auch noch einen instruktiven weiteren Fall, der sich an jene in der Dichtung vom Phäakenpalast sehr gut anschliesst.

Der dem 'homerischen' Demeterhymnus vorausliegenden Orpheusdichtung gehört ja auch der bekannte Passus an "solange sie noch die Erde und den gestirnten Himmel sah, hoffte sie noch ihre Mutter zu sehen", wobei der Lykomide bei Schaffung seines homerischen Kleinepos diesen Passus wohl übernimmt, ihn jedoch wenig sinnvoll erweitert (und dabei durch gleich zweimalige Vernachlässigung des Digamma sein Eingreifen - d.h. seine Version als die Sekundärversion- verrät):

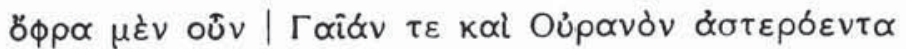

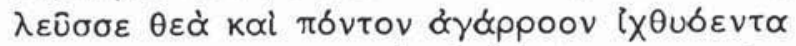

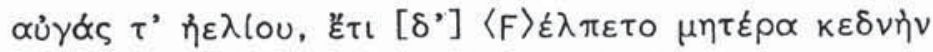

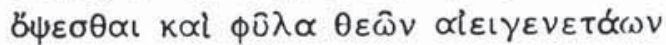

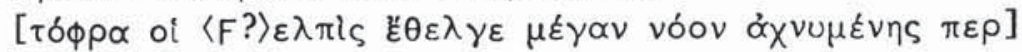

(Orph. fr. 49, 71 ff. = 'Hom.' 33 ff. $)^{81}$. Der Anfangsvers dieses Passus von Orpheusdichtung wäre auch in sich allein als altes Element der orpheischen Muse kenntlich, da die dem Hesiod bereits geläufige Wendung sich auch in der Orpheustradition mehrfach erhalten hat ${ }^{82}$. Er ist auch dem lykomidischen Letzten Dichter sehr wohl geläufig: in seiner Ilias verwendet er ihn viermal, wovon zweimal gleichartig (Il. V 769

\footnotetext{
79 Die Identität des Letzten Dichters an Ilias und Odyssee erweist ja schon die enge Zusammengehörigkeit von Iliasproömium und Nekyia, s. eingangs, dazu auch VdM, oben Anm. 34.

80 Für diese s. Peisistratos, S. 36 f. bezw. Orpheus, S. 150.

81 Dazu Peisistratos, S. 18 f. mit 19, 1.

82 Die Stellen bei Hesiod -Th. 45, 147, 154, 421, 427, 463, 470, 644, 702, Erg. 548zeigen (bei selbstverständlich leichter Variabilität) die auch dem Böoter schon überkommene poetische Wendung. - Orph., fr. $21,2=21 \mathrm{a}, 3,32 \mathrm{a}, 6,32 \mathrm{~b}, 3$ (57, 3), 168, 4 H. Hom. 13, 6, 14, 10, 37, 1. - Vgl. Peisistratos, SS. 34 und 44. - Ubrigens ist der ganze Passus nach der Methode des 'Fundamentalschlusses' -also unabhängig von der Bezeugung durch den Berliner Papyrus- als Orpheusdichtung aufweisbar: Orpheus, SS. 89-91.
} 
und VIII 46) ${ }^{83}$. Im Il. VIII, das ganz ihm gehört und demzufolge fast ganz aus Erborgtem gezimmert ist ${ }^{84}$, lässt er den Zeus seinen Reisewagen besteigen, um nach dem Ida zu gelangen. Der Göttervater treibt die Rosse an,

$\tau \dot{\omega} \delta^{\prime}$ oủk $\alpha^{\prime} \varepsilon \dot{\varepsilon} \kappa \nu \tau \varepsilon \pi \varepsilon \tau \dot{\varepsilon} \sigma \theta \eta \nu$

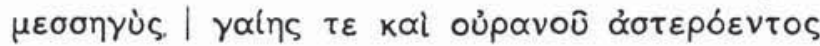

(VIII 45 f.). «Mitten zwischen Himmel und Erde dahinfliegen»: das überkommene poetische Element ist hier profan-meteorologisch verwendet, sodass sein religiös-mythischer Ursprung (der bei Hesiod und in den 'orphischen' Literaturpoesien spürbar bleibt) und namentlich der Ursprung aus dem Demetermythus völlig verwischt und vergessen ist, Himmel und Erde als Göttermächte nicht einmal mehr vorstellbar sind ${ }^{85}$. Diese Angabe der aviatischen Höhenposition des fliegenden Reisewagens ist wieder Profanation der gleichen Art wie die der besprochenen musischen Elemente in der Dichtung vom Alkinoospalast. Auch hier ist es des Orpheus Demeterdichtung, die dem Letzten Dichter aus dem lykomidischen Gentilerbe so geläufig ist, dass sie ihm unversehens zum poetischen Steinbruch wird, dem er sein homerisches Fabulieren verdankt ${ }^{* 6}$.

Gerade das ist auch noch an einer andern Stelle der Ilias offenkundig. Im Fünften Buch tadelt Sarpedon Hektor, dass er nicht kämpfe, worauf der Getadelte sich zum Kampfe bereit macht und auch das Heer zum Kämpfen antreibt. Die Troer setzen sich in Bewegung und treten den Achäern entgegen. Die aber halten Stand und fürchten sich nicht:

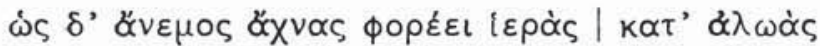

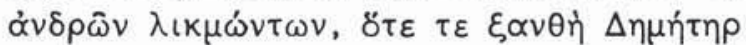

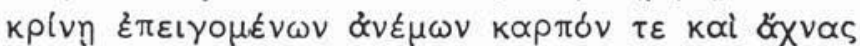

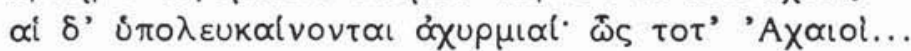

83 Auch. Il. V $769=$ "B" nach VdM, S. 98 ff. Ihm gehören auch die weiteren Stellen, an denen dies poetische Element verwendet ist: Il. XIV 174, Od. I 54, H. Ap. 334, sowie der Götterschwur Il. XV 36-38 = Od. V 184-86 = H. Ap. 84-86. (Zum Teilelement oủp. dor. s. Orpheus, S. 90, 3).

84 Leaf, Introd. to Il. VIII, VdM, Hyp., S. 144 f., Peisistratos, SS. 27-29.

85 Die Herausgeber drucken im Hesiod und 'Orpheus' Gaia und Uranos an den meisten Stellen mit Majuskel-Beginn: wäre das in Il. VIII 46 usw. vorstellbar? Ubrigens ist wohl auch das zweite Hemistich von VIII $45(=\mathrm{V} 366)$ gleichen Ursprungs: H. Dem. 379, und die Aussage erinnert ja auch sehr an Schamanenrosse.

8o Es geht natürlich nicht an, einfach von 'Formeln' zu sprechen: denn solche sind zu keiner Zeit vom Himmel gefallen. Aber einprägsame poetische Fügungen

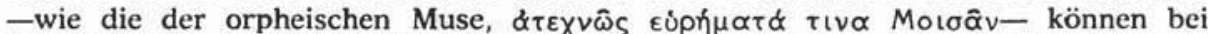
den Epigonen zu 'Formeln' erstarren und dann auch profan verwendet werden. 
(Il. V 499 ff.). Die entsprechende Orpheusdichtung hat sich in 'orphischer' Literatur bis in späte Zeit erhalten:

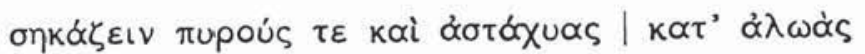

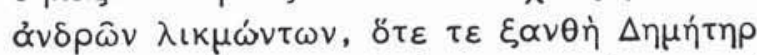

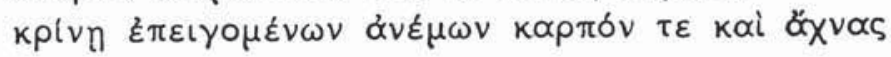

(Orph. fr. 268). «Weizen und Spreu gesondert sammeln, wenn auf den Tennen die Männer worfeln, und wenn die blonde Demeter bei den (die Spreu) treibenden Winden die Frucht und die Hülsen scheidet". Die wörtliche Ubereinstimmung zwischen 'Homer' und 'Orpheus' ist beachtlich: es ist die umfangreichste Ubereinstimmung, die es überhaupt gibt ${ }^{87}$. In der Ilias bildet diese Dichtungspartie ein Gleichnis für das Standhalten der Achäer, und zwar in einem Zusammenhang, der schon immer vielfach beanstandet oder gar athetiert wurde. Es ist «ein in die Erzählung sehr unvermittelt eintretendes" und "wegen seiner unbeholfenen und schiefen Anschauung mit Grund getadeltes Gleichnis» 88 , das sich auch noch unmittelbar an eine früher mehrheitlich verworfene Partie anschliesst ${ }^{89}$. Nun lässt sich aber durch keine irgendwie geartete Athetese Remedur schaffen: das Ganze ist ein Produkt des poetisch sehr unselbständigen Letzten Dichters, denn «Sarpedon ist überall erst vom Dichter B zugetan ${ }^{90}$. In dem von ihm geschaffenen Zusammenhang überrascht es nicht, dass die dem Gleichnis unmittelbar vorangehende Versgruppe, 494-498, aus dem Il. XI wörtlich übernommen ist (XI 211-214) ${ }^{91}$ ), und das nicht nur hier sondern ebenso auch in It. VI 103-106, wobei der letzte Vers noch ein weiteres Mal benutzt ist, It. XVII 343. Dieses wörtliche Übernehmen -bei mechanischem Abschreiben von schriftlichen Vorlagen - ist die Art des Letzten Dichters mit homerischer Dichtung umzugehen ${ }^{92}$. Ist aber das vorangehende Stück keine originale Dichtung, so kann das dazugehörige Gleichnis ebenfalls keine solche sein: also sind die 'Orpheus'-Verse wirklich keine 'homerische' Originalschöpfung, die es erlaubte, ein 'Orphikerplagiat aus Homer' zu statuieren. Der Fall erinnert vielmehr an das Il. VIII, wo gleichfalls just im Zusammenhang unselbständigen und übernom-

\footnotetext{
87 Umso erstaunlicher, dass bei Kern unter den «Epici ab Orphicis adhibiti» (Index V, p. 398) dieser Fall $\mathrm{nicht}$ verzeichnet ist.

88 So Ameis-Hentze, Il. erklärt, Anhang 22, S. $75 \mathrm{f}$.

89 Die vv. 471-96 wurden von überaus zahlreichen früheren Philologen athetiert, s. bei Ameis-H., a. O., S. 72.

90 So VdM, Hyp., S. 99 , dazu auch a. O. 247-49.

91 So ausdrücklich VdM, S. 193.

92 Dies auf Grund früherer analytischer Literatur kurz zusammengefasst in Peisistratos, SS. 27-29.
} 
menen Dichtens Orpheusverse begegnen, z.B. VIII 19 f., wo also der Lykomide im Rahmen des aus Homeridendichtung Erborgten auch von seiner orpheischen Muse Gebrauch macht ${ }^{93}$. Weiss man um die Orpheustradition seines Geschlechts, so kann es überhaupt keinen Zweifel geben, dass der Letzte Dichter in der V-Partie zweierlei aneinandergeschoben hat: den dem Il. XI entnommenen Passus aus alter Homeridendichtung und den aus der ihm geläufigen demetrischen Orpheusdichtung, weshalb das Gleichnis denn auch "sehr unvermittelt» in die Erzählung eintritt.

Und dieser Sachverhalt bestätigt sich auch in einigen nicht zu übersehenden Einzelheiten. In der Klausel $\xi \alpha v \theta \dot{\eta} \Delta \eta \mu \dot{\tau} \tau \eta \rho$ hat diese Dichtung eine Exclusiv-Parallele zum 'homerischen' -d.h. vom Dichter selbst geschaffenen- Demeterhymnus (v. 302): und das ist ja -zumal als Element der dem Hymnus zu Grunde liegenden orpheischen Musewie alle derartigen Parallelen als Zeugnis der Dichter-Identität zu nehmen ${ }^{94}$. Hinzu kommt, dass der Anfang der als Gleichnis benutzten Partie abgeändert ist, und zwar ersichtlich ausgehend von der vorgegebenen Orpheusdichtung: daher sind zwei Wiederholungen hereingekommen,

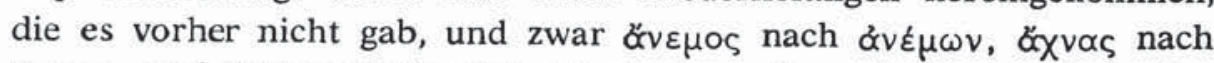

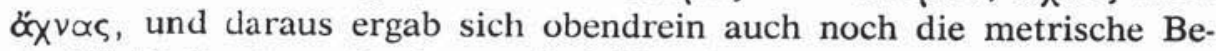
denklichkeit, dass die kurze Endsilbe des neuen «̋veros nun Arsis bildet ${ }^{95}$. Die Verwendung des Passus als Gleichnis im 'Homer' ist also offensichtlich sekundär gegenüber der Orpheusdichtung: Demetrisches ist ja eigentlich homerfremd und nur durch den Letzten Dichter hereingebracht ${ }^{*}$. Schliesslich auch dies: die Verwendung dieser demetrischen Orpheusdichtung als blosses Gleichnis für eine Kampfszene ist zweifelsohne auch wieder ein Schritt in der Richtung auf die Profanation der alten Muse.

Von hier aus läge bereits der Schluss auf die Proekthesis nahe, wo ja ebenfalls ein dem Demetrischen entstammender - und ebenfalls abgeänderter - Orpheusvers in gleicher Weise im Verein mit sekundär verwendeter Dichtung erscheint, und wo aus der Anderung gleichfalls eine metrische Bedenklichkeit resultiert ${ }^{97}$. Doch ist um der "Menis" willen zuvor noch ein weiterer Fall heranzuziehen.

93 Peisistratos, SS. $35-37$.

94 Peisistratos, SS. 41-60, die Demeter-Klausel $50 \mathrm{f}$.

95 Metrische Bedenklichkeiten in der Sekundärdichtung des Letzten Dichters: H. Dem. 54 ff. gegenüber Orph., fr. 49,102 ff. (Peisistratos, S. 20 f.), H. Dem, 33-37 gegenüber Orph., fr. 49, 69 ff. (Peisistratos, S. 19, 1), Il. XX 61 gegenüber H. Dem. 357 (Peisistratos, S. 53).

\% Vgl. Od. V 125-28, oben Anm. 69. Zu Il. II 696 und XIV 326 s. VdM.

97 Der antike Zeuge Iustin moniert diese (s. Orph., fr. 48 K.), womit er im 
Mythus und Dichtung von Demeter kennen von Anfang an als ursprüngliches Motiv und Kern ihrer heiligen Geschichte den Z or n der Göttin, der nach dem Raube ihrer Tochter sie bestimmt ${ }^{98}$. Grollend sitzt sie fern von den Göttern (303 ff.), erzürnt hemmt sie das Wachstum und hätte damit beinahe das Menschengeschlecht vertilgt und die Olympier um die Opfer gebracht. Ihr Zürnen ist so nachhaltig und unerbittlich, dass Zeus zuerst die Iris zu ihr schickt, erfolglos, dass er danach alle Götter schickt, erfolglos: erst wenn sie ihre Tochter wieder sehe, wolle sie den Olymp wieder betreten und die Erde wieder Frucht emporsenden lassen (314-333). Darauf schickt Zeus schliesslich den Götterboten zum Hades, mit dem Auftrag, diesen zu bereden und Persephone wieder ans Licht emporzuführen, ö $\phi \rho \alpha \varepsilon \mu \eta \tilde{\eta} \rho \rho \delta \phi \theta \alpha \lambda \mu \circ \hat{\imath} \sigma \iota[v]$

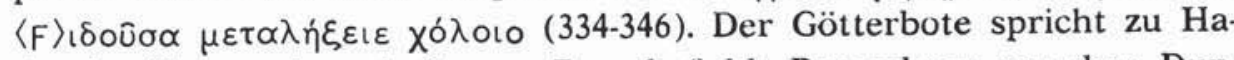
des im Sinne seines Auftrags: Zeus befiehlt Persephone aus dem Dunkelreich wieder emporzusenden $\not \iota \phi \rho \alpha \hat{\varepsilon} \mu \eta \dot{\tau} \eta \rho$

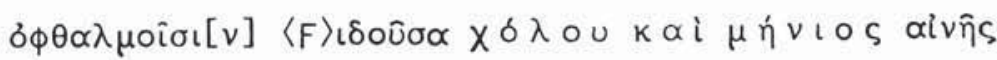

$$
\begin{aligned}
& \alpha \theta \propto \nu \alpha \tau \text { \& } \theta \pi \propto u ́ \sigma \varepsilon เ \varepsilon v
\end{aligned}
$$

(349 ff.). Des Zeus Wille geschieht, und die mit ihrer Mutter wieder Vereinigte berichtet ihr, dass Hermes im Auftrag des Kroniden gekommen sei, sie emporzuholen

$$
\begin{aligned}
& \text { iv } \propto \mu^{\prime} \delta \phi \theta \alpha \lambda \mu \circ i \tilde{\sigma}[v]\langle F\rangle
\end{aligned}
$$

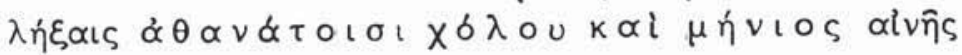

(409 f.). Ist schon das Grundmotiv des Demetermythus und =hymnus der Göttin «Menis», so lässt die poetische Form in der leicht variierenden Wiederholung erkennen, dass auch sie vom Lykomiden bei Schaffung seines 'homerischen' Hymnus als tradierte Prägung übernommen ist, auch sie natürlich aus der musisch-poetischen Orpheustradition, die ihm als Gentilerbe vertraut war ${ }^{99}$. Und auch dieses poetische

Blick auf H. Dem. 55 gegenüber Orph., fr. 49, 103 offensichtlich ernst zu nehmen ist, s. vorletzte Anm.

98 Das Zorn-Motiv durchzieht das Ganze: H. Dem. 83, 91, 303 ff., 329 f., 338 f., 349 f., 354, 409 f., 452 f., 467 f.

99 'In entscheidenden Teilen ist ja der Ursprung des 'homerischen' Hymnus aus der Orpheustradition unabhängig vom Pap. Berol. schon aufgewiesen, Orpheus, SS. 76-111, auch oben S. 204, sodass es also - angesichts des desolaten Zustands des von älterer 'Orpheus'-Dichtung Uberlieferten - nichts besagt, wenn das in die 'homerische' Version übernommene $\& \theta \alpha v . x . \kappa . \mu$. diesmal rein zufällig nicht auch als Orpheusdichtung bezeugt ist. - Die attische Genetivform $\chi \delta \lambda^{\circ}$ ou in 350 und 410 ist natürlich junges Substitut, etwa für älteres $\chi \delta \lambda \circ \iota^{\circ} 1 \delta \dot{\varepsilon}$. Dass der Lykomide Alteres oftmals in attische Formen abgeändert oder in Alterem attische Formen eingefügt hat, ist mehrfach zu beobachten, z. B. im misogynen Vers oder oben S. 224 das ovias. 
Kernelement hat er in einer seiner Iliaspartien wörtlich wiederverwendet:

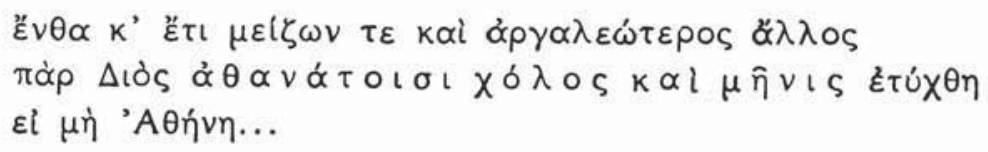

(Il. XV $121 \mathrm{ff}.)^{100}$. Auch hier ist die Sekundärverwendung des orpheischdemetrischen Elements nicht zu verkennen. Zeus war nach der «Dios Apate» beim Erwachen aufgebracht und ungehalten, natürlich gegenüber seiner Gemahlin, mit der er sich auseinandersetzt (Il. XV 1 ff., bes. 14 ff.). Da sich dann Ares gegen das Gebot des Zeus eigenmächtig zu den Achäern begeben will und sich dazu rüstet, wäre Zeus "gegen die Unsterblichen noch aufgebrachter gewesen, hätte nicht Athene eingegriffen und ihn zurückgehalten». In diesem irrealen Gedankengefüge, einem momentanen Einfall, der das Eingreifen Athenes vorbereiten soll, wird niemand den Ursprung des im Demetermythus zentralen Menis-Motivs sehen wollen. Die Identität der poetischen Wendung zeigt aber die Unabtrennbarkeit des Einen vom Andern. Folglich liegt auch hier das Umgekehrte vor: die Verwendung der vertrauten poetischen Fügung vom Groll der Demeter im Dienste eines ziemlich banalen Gedankens in einem ausserdemetrischen Zusammenhang. Und man wird, vergleichend, wohl auch noch Folgendes sehen: Demeter hielt sich in ihrem Groll von alle n Unsterblichen fern (v. 303), Zeus schickte a ll e Götter, um sie umzustimmen (326), durch die Wiederkehr ihrer Tochter solle ihr Groll gegenüber a lle n Unsterblichen ein Ende nehmen (351,

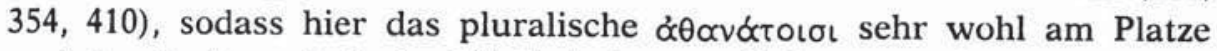
und durch die mehrfache Wiederholung auch fest verankert ist. In der Situation nach der 'Dios Apate' müsste Zeus ausser seiner raffinierten Gemahlin eigentlich nur den seinem Willen zuwider Handelnden (also etwa Poseidon und dem eigenmächtigen Ares) zürnen: eine Verschlimmerung des Zorns gegenüber 'den' Unsterblichen kommt unvorbereitet und erklärt sich aus der Applikation der dem Dichter geläufigen poetischen Wendung, die ihm dann auch noch den -erläuternd nachfolgenden-Gedanken eingegeben haben dürfte, dass Athene eingreift, weil sie in der gegebenen Situation für 'alle' Götter fürchtet. Der Dichter hat also auch hier das ihm vertraute mythisch-poetische Kernelement vom Groll der Demeter ursprungsfremd verwendet.

100 Gehört «B» nach VdM, S. 227 ff. 
Und das Gleiche tat er ebenso auch zur Eröffnung seiner Ilias, wo er den Orpheus-Vers von der Demeter Menis

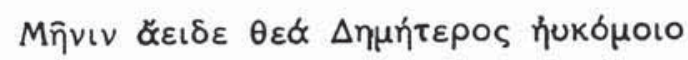

bezw.

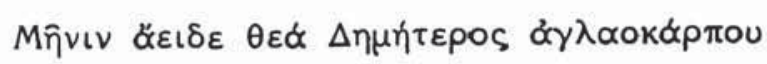

(er kannte ja beides) zum Eröffnungsvers vom Groll des Peliden machte:

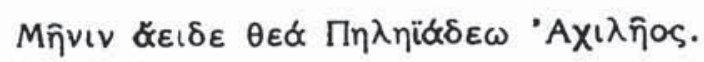

Dieser Rückgriff auf einen Orpheus-Vers reiht sich ein in die andern Beispiele der zu beobachtenden Dichtmanier, dass der Letzte Dichter altes poetisches Gut der orpheischen Muse aus der Assoziation des Demetermythus benutzt und profan in neuartigen Zusammenhängen verwendet. In keinem der besprochenen Fälle liesse sich die Abhängigkeit umkehren. Das von ihm aufgegriffene Traditionsgut ist eben jenes Orpheus-Erbe, das den Lykomiden als altes Gentilvermächtnis zu eigen war und als solches dem Letzten Dichter ja auch Grundlage und Anregung zu seinem 'homerischen' Kleinepos wurde.

Und nun ist zusammenzunehmen:

- Mit Il. I 1 verhält es sich nicht anders als mit den andern Entlehnungen und Profanierungen mythisch-poetischer Elemente der Orpheustradition aus dem Bereiche des Demetrischen, die sich in Partien des Letzten Dichters finden.

- Mit Il. I 1 verhält es sich nicht anders als mit den übrigen Elementen junger und sekundärer Dichtung innerhalb des sogenannten 'Proömium' des Grossepos: dem adverbialen $\tau \grave{\alpha}$ $\pi \rho \hat{\omega} \tau \alpha$, der den Kyprien entlehnten Ratschlussformel, dem die $\kappa \varepsilon \phi \propto \lambda \alpha$ l durch die $\psi \cup \chi \alpha \propto l$ ersetzenden Vers, den mehrfachen Beziehungen zur Kirke-Nekyia-Dichtung des Letzten Dichters.

Diese beiden Gegebenheiten ergänzen und stützen sich gegenseitig. Und dem ist dann als Drittes noch anzufügen:

- Mit der Uberlieferung zu Il. I 1, die von der Priorität des Orpheus spricht - bei Justin und Tzetzes - verhält es sich nun nicht anders als mit den übrigen inzwischen bereits gerechtfertigten Uberlieferungen von des Orpheus Priorität: so vor allem derjenigen zu It. V 499-501 (Orph. fr. 268), ein Parallelfall, in dem auch Tzetzes - sogar als einziger Zeuge- gerechtfertigt ist, sowie mit den Uberlieferungen zu Il. XX 308 (Orph. fr. 4), Od. XI 427 (Orph. fr. 234), XXIV 1 (s. Kern LIV, $2.0-4$ 
test. 97, vgl. Orph. fr. 233, 7), H. Dern. (Orph. fr. 49) -um hier nur die wichtigsten zu nennen-, die inzwischen ebenfalls nicht mehr in Zweifel gezogen werden können ${ }^{101}$.

Diesem Ergebnis kann nun noch eine weitere Beobachtung angefügt werden. Die 'Menis' der ersten Vershälfte ist seit alters der 'Götterzorn', der tief wurzelnde lang anhaltende Groll aus dem Reiche der Unsterblichen, besonders auch der Unterirdischen. Das Wort gehört daher der sakralen Sphäre an, was sich formell schon darin ausdrückt, dass beispielsweise auch Platon noch den Genetiv $\mu$ ŕvıos gebraucht ${ }^{102}$. Daher ist auch im Epos das Wort an einer erdrückenden Mehrzahl von Stellen vom Zürnen eines Gottes gebraucht ${ }^{103}$, während die Ubertragung auf den Sterblichen, den Helden, vom lykomidischen Dichter im Zuge der Adaptation der orpheischen poetischen Prägung vorgenommen wurde und sich daher in diesem Sinne nur an wenigen Stellen eben dieses Dichters findet ${ }^{104}$. Und mit der vom Vorbild angeregten Neubildung $\Pi \eta \lambda \eta \ddot{i} \alpha \delta \varepsilon \omega$ 'AXı $\lambda \hat{\eta}$ os fügt sich der 'homerische' Menisvers auch zu den fünf andern Stellen der Ilias und den beiden Odysseestellen in grosser und kleiner Nekyia, wo der lykomidische Dichter diese seine Eigenprägung wiederholt hat ${ }^{105}$. Die beiden Vershälften gehören aber zusammen: bei diesem Befund - der Ubertragung des Götterzorns auf einen Sterblichen bei klanganaphorischer Nachbildung des zweiten Hemistichs - das Verhältnis der beiden 'Menisverse' umkehren zu wollen, ist unmöglich.

Schlussendlich steht das Resultat nicht nur im Einklang mit der speziellen Uberlieferung, wonach 'Homer' im Menisvers 'Orpheus' folge, sondern auch mit der allgemeinen Uberzeugung des gesamten Griechen tums, wonach Orpheus lange vor Homer gelebt und gesungen hat ${ }^{106}$.

101 Das ist bereits gezeigt: Orph., fr. 268: Peisistratos, S. 50 f. -Orph., fr. 4: Orpheus, S. 99 f. -Orph., fr. 234: Orpheus, S. 33 f., 98, bes. Sänger d. Vz., SS. 26-29 mit 39-49. - Od. XXIV 1: Orpheus, SS. 164-68, 224 f., 233 f.-Orph. fr. 49: Orpheus, SS. 101-111 (u. passim), Peisistratos, SS. 16-21.

102 E. Schwyzer, Rh. Mus. 80, 1931, S. 213 ff. Normal-attisches $\mu \eta \dot{v} v \varepsilon \omega \varsigma$, das man bei einem Profanbegriff erwarten müsste, ist nicht belegt.

103 Il. I 75, V 34, 178, 444, XIII 624, XV 122, XVI 711, XXI 523, Od. II 66, III 135 V 146, XIV 283. H. Aphr. 290.

104 Nur 17 . IX 517, XIX 35 und 75. - Auch das Verbum $\mu \eta v i \omega$ ist stets nur in Partien des Letzten Dichters gebraucht: Il. I 247, 422, 488, II 769, 772, VII 230 , IX 426, XII 10, XIII 460, XVIII 257, XIX 62 (dazu jeweils VdM a. O., zu Il. I 422 zwar nicht auf S. 26 f., wohl aber 29 und 31), und es hat teilweise attische Form, Wackernagel, Sprachl. Unters. zu Hom., S. 140.

105 S. oben S. 213 f. mit Anm. 40.

106 "To fly in the face of so universal a tradition is an audacious act", W. K. C. Guthrie, Gnomon 26, 1954, S. 304. - Orpheus als Mykenäer : s. oben S. 204. 
Und diese ist inzwischen angesichts der Bedeutung des Sängers im Neleischen Pylos gut gesichert. Das Verhältnis der beiden Grossen zueinander umkehren $\mathrm{zu}$ wollen, lässt sich heute durch nichts mehr rechtfertigen.

Das Vorgehen des Letzten Dichters, seine 'Techne', verdient ein Wort der Würdigung. In der alten Memnonis war es eine mütterliche Warnung, die den Achill sich des Kampfes enthalten liess. Homerisch wurde das Motiv dieser Enthaltung dann Streit und Groll: ěpıs und Xó $\lambda$ os, $\chi \circ \lambda \omega \theta \hat{\eta} \nu \propto \iota, \chi \propto \lambda \varepsilon \pi \hat{\eta} v \propto \iota, \chi \omega o ́ \mu \varepsilon v \circ \varsigma$. Im Blick auf diesen Groll hat dann der Letzte Dichter die göttliche Menis Demeters auf den Sterblichen übertragen: womit er das Zorn-Motiv so eindrücklich markiert hat, dass er es gut und bequem zum Strukturmotiv und d.h. zum Rückgrat des 'Iliadischen' Grossepos machen konnte. Das darf man, so einfach es ist, als genial bezeichnen, und der Erfolg gab ihm jedenfalls recht. Die Ubertragung und Profanierung solch alttradierter religiöser Poesie ist für die Menis ja nicht kühner und nicht schlimmer als für jene in der Dichtung vom Phäakenpalast verwendeten Elemente demetrischer Dichtung oder als der Vers von Gaia und Ouranos zur Bezeichnung der aviatischen Höhenposition des fliegenden Reisewagens. Das kühne Neuern war in jenem Umbruch am Ende des epischen Zeitalters möglich, schon ein Hesiod -es sei an seinen Negervers erinnert- ist davon nicht frei.

Das Problem der beiden Menisverse findet seine Lösung wenn der Letzte Dichter als Lykomide identifiziert und die musisch-poetische Gentiltradition dieses sich vom Neleischen Pylos herleitenden Priestergeschlechts beachtet wird.

ROBERT BÖHME 